\title{
MIURA OPERS AND CRITICAL POINTS OF MASTER FUNCTIONS
}

\author{
EVGENY MUKHIN ${ }^{*, 1}$ AND ALEXANDER VARCHENKO**,2
}

\begin{abstract}
Critical points of a master function associated to a simple Lie algebra $\mathfrak{g}$ come in families called the populations MV1. We prove that a population is isomorphic to the flag variety of the Langlands dual Lie algebra ${ }^{t} \mathfrak{g}$. The proof is based on the correspondence between critical points and differential operators called the Miura opers.

For a Miura oper $D$, associated with a critical point of a population, we show that all solutions of the differential equation $D Y=0$ can be written explicitly in terms of critical points composing the population.

\author{
* Department of Mathematical Sciences, \\ Indiana University Purdue University Indianapolis, \\ 402 North Blackford St., Indianapolis, IN 46202-3216, USA \\ ${ }^{* *}$ Department of Mathematics, University of North Carolina at Chapel Hill, \\ Chapel Hill, NC 27599-3250, USA
}

\section{INTRODUCTION}

In $[\mathrm{ScV}]$ rational functions were considered which are products of powers of linear functions. It was discovered that under certain conditions all critical points of the rational functions are non-isolated and form non-trivial varieties. It is not clear yet how general that phenomenon is but the phenomenon certainly holds for products of powers of linear functions appearing in representation theory. Those products are called the master functions.

Let $\mathfrak{h}$ be the Cartan subalgebra of a simple Lie algebra $\mathfrak{g} ;\left(\right.$, , ) the Killing form on $\mathfrak{h}^{*}$; $\alpha_{1}, \ldots, \alpha_{r} \in \mathfrak{h}^{*}$ simple roots; $\Lambda_{1}, \ldots, \Lambda_{n} \in \mathfrak{h}^{*}$ dominant integral weights; $l_{1}, \ldots, l_{r}$ nonnegative integers; $z_{1}, \ldots, z_{n}$ distinct complex numbers. The master function $\Phi$ associated with this data is given by formula (II). It is a rational function of $l_{1}+\cdots+l_{r}$ variables $t_{1}^{(1)}, \ldots, t_{l_{1}}^{(1)}, t_{1}^{(2)}, \ldots, t_{l_{r}}^{(r)}$, and $n$ variables $z_{1}, \ldots, z_{n}$. We may think that $l_{1}+\cdots+l_{r}+n$ particles are given in $\mathbb{C}$. The particle $t_{j}^{(i)}$ has weight $-\alpha_{i}$ and the particle $z_{s}$ has weight $\Lambda_{s}$. The particles interact pairwise. The interaction of particles $x$ and $y$ with weights $v$ and $w$, respectively, is given by $(x-y)^{(v, w)}$. Then total interaction is the product of such terms over the set of all pairs. The master function describes the interaction of $t$-particles and $z$-particles.

${ }^{1}$ Supported in part by NSF grant DMS-0140460.

2 Supported in part by NSF grant DMS-0244579. 
The master function appears in hypergeometric solutions to the $\mathrm{KZ}$ equations with values in the tensor product of irreducible highest weight representations $L_{\Lambda_{1}}, \ldots, L_{\Lambda_{n}}$ with highest weights $\Lambda_{1}, \ldots, \Lambda_{n}$, respectively. The solutions have the form

$$
u(z)=\int \Phi(\boldsymbol{t} ; \boldsymbol{z})^{1 / \kappa} A(\boldsymbol{t} ; \boldsymbol{z}) d \boldsymbol{t},
$$

where $\kappa$ is the parameter of the $\mathrm{KZ}$ equations and $A(\boldsymbol{t} ; \boldsymbol{z})$ is some explicitly written rational function with values in the tensor product $\mathrm{SV}$.

The master function also appears in the Bethe ansatz of the Gaudin model with values in the same tensor product $[\mathrm{RV}]$. In that case the value of the function $A(\cdot ; \boldsymbol{z})$ at a point $\boldsymbol{t}$ is an eigenvector of the commuting Gaudin Hamiltonians if $\boldsymbol{t}$ is a critical point of the master function.

In this paper we study critical points of the master function on the set where all $\left\{t_{j}^{(i)}, z_{s}\right\}$ are distinct. In other words we study those positions of distinct particles $\left\{t_{j}^{(i)}\right\}$ in the complement to $\left\{z_{s}\right\}$ which extremize the master function.

Critical points of master functions associated to a simple Lie algebra $\mathfrak{g}$ come in families called populations $[\mathrm{ScV}, \mathrm{MV} 1$. In this paper we prove that a population is isomorphic to the flag variety of the Langlands dual Lie algebra ${ }^{t} \mathfrak{g}$. The proof is based on the correspondence between critical points and differential operators called the Miura opers.

To every critical point $\boldsymbol{t}$ one assigns a certain linear differential operator $D_{\boldsymbol{t}}$ with coefficients in ${ }^{t} \mathfrak{g}$, called the Miura oper. The differential operators of that type were considered by V. Drinfeld and V. Sokolov in their study of the KdV type equations [DS]. On opers and Miura opers see [BD, FFR, F1, F2, MV1, BM, MV4.

Different critical points correspond to different Miura opers. The Miura opers corresponding to critical points of a given population form an equivalence class with respect to suitable gauge equivalence. We show that the equivalence class of Miura opers is isomorphic to the flag variety of ${ }^{t} \mathfrak{g}$.

In [MV1, BM] we considered Miura opers for Lie algebras of types $A_{r}, B_{r}, C_{r}, G_{2}$ and using the opers proved that a population of critical points of types $A_{r}, B_{r}, C_{r}, G_{2}$ is isomorphic to the corresponding flag variety. The proof, suggested in the present paper, is more direct and works for any simple Lie algebra.

If $D_{\boldsymbol{t}}$ is the Miura oper corresponding to a critical point $\boldsymbol{t}$, then the set of solutions of the differential equation $D_{t} Y=0$ with values in a suitable space is an important characteristics of the critical point. We used that characteristics for $s l_{r+1}$ Miura opers in MV1 to give a bound from above for the number of populations of critical points of the corresponding $s l_{r+1}$ master function. That statement in MV1 was in some sense opposite to the Bethe ansatz conjectures, see [MV1].

It turns out that for any simple Lie algebra $\mathfrak{g}$ and any critical point $\boldsymbol{t}$ of a $\mathfrak{g}$ master function all solutions of the differential equation $D_{\boldsymbol{t}} Y=0$ can be written explicitly in terms of critical points composing the population originated at $\boldsymbol{t}$. Thus the population of critical points "solves" the Miura differential equation $D_{t} Y=0$. This is the second main result of the paper. 
When this paper was being written preprint F2 by E. Frenkel appeared. The preprint is devoted to the same fact that the variety of gauge equivalent ${ }^{t} \mathfrak{g}$ Miura opers is isomorphic to the flag variety of ${ }^{t} \mathfrak{g}$. The main result of [F2 is Corollary 3.3. In our opinion the proof of Corollary 3.3 in [F2] is incomplete and in particular the statement of Corollary 3.3 is not correct.

The idea of this paper was originated in discussions with E. Frenkel in the spring of 2002. As a result of those discussions two papers appeared: this one and F2.

We thank E. Frenkel for stimulating meetings which originated this paper. We thank P. Belkale and S. Kumar for numerous useful discussions.

The paper is organized as follows. In Section 2 we introduce populations of critical points. In Section 3 we discuss elementary properties of Miura opers corresponding to critical points. In Section 4 we prove that the variety of gauge equivalent Miura opers is isomorphic to the flag variety, see Theorem 4.2. We discuss the relations between the Bruhat cell decomposition of the flag variety and populations of critical points in Section 5. The main result there is Corollary 5.3 describing the structure of connected components of the critical set of master functions. In Section 6 we give explicit formulas for solutions of the differential equation $D_{t} Y=0$, see Theorems 6.1, 6.2, 6.3,

\section{Master functions AND CRItical points, MV1}

2.1. Kac-Moody algebras. Let $A=\left(a_{i, j}\right)_{i, j=1}^{r}$ be a generalized Cartan matrix, $a_{i, i}=2$, $a_{i, j}=0$ if and only $a_{j, i}=0, a_{i, j} \in \mathbb{Z}_{\leq 0}$ if $i \neq j$. We assume that $A$ is symmetrizable, i.e. there exists a diagonal matrix $D=\operatorname{diag}\left\{d_{1}, \ldots, d_{r}\right\}$ with positive integers $d_{i}$ such that $B=D A$ is symmetric.

Let $\mathfrak{g}=\mathfrak{g}(A)$ be the corresponding complex Kac-Moody Lie algebra (see [K], §1.2), $\mathfrak{h} \subset \mathfrak{g}$ the Cartan subalgebra. The associated scalar product is non-degenerate on $\mathfrak{h}^{*}$ and $\operatorname{dim} \mathfrak{h}=r+2 d$, where $d$ is the dimension of the kernel of the Cartan matrix $A$.

Let $\alpha_{i} \in \mathfrak{h}^{*}, \alpha_{i}^{\vee} \in \mathfrak{h}, i=1, \ldots, r$, be the sets of simple roots, coroots, respectively. We have

$$
\begin{aligned}
\left(\alpha_{i}, \alpha_{j}\right) & =d_{i} a_{i, j}, \\
\left\langle\lambda, \alpha_{i}^{\vee}\right\rangle & =2\left(\lambda, \alpha_{i}\right) /\left(\alpha_{i}, \alpha_{i}\right), \quad \lambda \in \mathfrak{h}^{*} .
\end{aligned}
$$

In particular, $\left\langle\alpha_{j}, \alpha_{i}^{\vee}\right\rangle=a_{i, j}$.

Let $\mathcal{P}=\left\{\lambda \in \mathfrak{h}^{*} \mid\left\langle\lambda, \alpha_{i}^{\vee}\right\rangle \in \mathbb{Z}\right\}$ and $\mathcal{P}^{+}=\left\{\lambda \in \mathfrak{h}^{*} \mid\left\langle\lambda, \alpha_{i}^{\vee}\right\rangle \in \mathbb{Z}_{\geq 0}\right\}$ be the sets of integral and dominant integral weights.

Fix $\rho \in \mathfrak{h}^{*}$ such that $\left\langle\rho, \alpha_{i}^{\vee}\right\rangle=1, i=1, \ldots, r$. We have $\left(\rho, \alpha_{i}\right)=\left(\alpha_{i}, \alpha_{i}\right) / 2$.

The Weyl group $\mathcal{W} \in \operatorname{End}\left(\mathfrak{h}^{*}\right)$ is generated by reflections $s_{i}, i=1, \ldots, r$,

$$
s_{i}(\lambda)=\lambda-\left\langle\lambda, \alpha_{i}^{\vee}\right\rangle \alpha_{i}, \quad \lambda \in \mathfrak{h}^{*} .
$$

We use the notation

$$
w \cdot \lambda=w(\lambda+\rho)-\rho, \quad w \in \mathcal{W}, \lambda \in \mathfrak{h}^{*},
$$


for the shifted action of the Weyl group.

The Kac-Moody algebra $\mathfrak{g}(A)$ is generated by $\mathfrak{h}, e_{1}, \ldots, e_{r}, f_{1}, \ldots, f_{r}$ with defining relations

$$
\begin{aligned}
{\left[e_{i}, f_{j}\right] } & =\delta_{i, j} \alpha_{i}^{\vee}, \quad i, j=1, \ldots r, \\
{\left[h, h^{\prime}\right] } & =0, \quad h, h^{\prime} \in \mathfrak{h}, \\
{\left[h, e_{i}\right] } & =\left\langle\alpha_{i}, h\right\rangle e_{i}, \quad h \in \mathfrak{h}, i=1, \ldots r, \\
{\left[h, f_{i}\right] } & =-\left\langle\alpha_{i}, h\right\rangle f_{i}, \quad h \in \mathfrak{h}, i=1, \ldots r
\end{aligned}
$$

and the Serre's relations

$$
\left(\operatorname{ad} e_{i}\right)^{1-a_{i, j}} e_{j}=0, \quad\left(\operatorname{ad} f_{i}\right)^{1-a_{i, j}} f_{j}=0
$$

for all $i \neq j$. The generators $\mathfrak{h}, e_{1}, \ldots, e_{r}, f_{1}, \ldots, f_{r}$ are called the Chevalley generators.

Denote $\mathfrak{n}_{+}\left(\right.$resp. $\left.\mathfrak{n}_{-}\right)$the subalgebra generated by $e_{1}, \ldots, e_{r}\left(\right.$ resp. $\left.f_{1}, \ldots, f_{r}\right)$. Then $\mathfrak{g}=\mathfrak{n}_{+} \oplus \mathfrak{h} \oplus \mathfrak{n}_{-}$. Set $\mathfrak{b}_{ \pm}=\mathfrak{h} \oplus \mathfrak{n}_{ \pm}$.

Let $\mathfrak{g}=\oplus_{j} \mathfrak{g}^{j}$ be the canonical grading of $\mathfrak{g}$. Here we have $e_{i} \in \mathfrak{g}^{1}, f_{i} \in \mathfrak{g}^{-1}, \mathfrak{n}_{+}=$ $\oplus_{j>0} \mathfrak{g}^{j}, \mathfrak{h}=\mathfrak{g}^{0}, \mathfrak{n}_{-}=\oplus_{j<0} \mathfrak{g}^{j}$

For a vector space $X$ we denote $M(X)$ the space of $X$-valued rational functions on $\mathbb{C}$.

We denote $\bar{M}\left(\mathfrak{n}_{+}\right)$the completion of the space $M\left(\mathfrak{n}_{+}\right)$with respect to the canonical grading. An element of $\bar{M}\left(\mathfrak{n}_{+}\right)$is a formal sum $\sum_{j>0} u_{j}$, where $u_{j}: \mathbb{C} \rightarrow \mathfrak{g}^{j}$ are rational functions.

The Kac-Moody algebra ${ }^{t} \mathfrak{g}=\mathfrak{g}\left({ }^{t} A\right)$ corresponding to the transposed Cartan matrix ${ }^{t} A$ is called Langlands dual to $\mathfrak{g}$. Let ${ }^{t} \alpha_{i} \in{ }^{t} \mathfrak{h}{ }^{*},{ }^{t} \alpha_{i}^{\vee} \in{ }^{t} \mathfrak{h}, i=1, \ldots, r$, be the sets of simple roots, coroots of ${ }^{t} \mathfrak{g}$, respectively. Then

$$
\left\langle{ }^{t} \alpha_{i},{ }^{t} \alpha_{j}^{\vee}\right\rangle=\left\langle\alpha_{j}, \alpha_{i}^{\vee}\right\rangle=a_{i, j}
$$

for all $i, j$.

2.2. The definition of master functions and critical points. We fix a Kac-Moody algebra $\mathfrak{g}=\mathfrak{g}(A)$, a non-negative integer $n$, a collection of dominant integral weights $\boldsymbol{\Lambda}=\left(\Lambda_{1}, \ldots, \Lambda_{n}\right), \Lambda_{i} \in \mathcal{P}^{+}$, and points $\boldsymbol{z}=\left\{z_{1}, \ldots, z_{n}\right\} \subset \mathbb{C}$. We assume that $z_{i} \neq z_{j}$ if $i \neq j$. We often do not stress the dependence of our objects on these parameters.

In addition we choose a collection of non-negative integers $\boldsymbol{l}=\left(l_{1}, \ldots, l_{r}\right) \in \mathbb{Z}_{\geq 0}^{r}$. The choice of $\boldsymbol{l}$ is equivalent to the choice of the weight

$$
\Lambda_{\infty}=\sum_{i=1}^{n} \Lambda_{i}-\sum_{j=1}^{r} l_{j} \alpha_{j} \in \mathcal{P} .
$$

The weight $\Lambda_{\infty}$ will be called the weight at infinity. 
The master function $\Phi\left(\boldsymbol{t} ; \Lambda_{\infty}\right)$ is defined by

$$
\begin{aligned}
& \Phi\left(\boldsymbol{t} ; \Lambda_{\infty}\right)=\Phi\left(\boldsymbol{t} ; \boldsymbol{z}, \boldsymbol{\Lambda}, \Lambda_{\infty}\right)=\prod_{1 \leq s<u \leq n}\left(z_{s}-z_{u}\right)^{\left(\Lambda_{s}, \Lambda_{u}\right)} \times \\
& \prod_{i=1}^{r} \prod_{j=1}^{l_{i}} \prod_{s=1}^{n}\left(t_{j}^{(i)}-z_{s}\right)^{-\left(\Lambda_{s}, \alpha_{i}\right)} \prod_{i=1}^{r} \prod_{1 \leq j<s \leq l_{i}}\left(t_{j}^{(i)}-t_{s}^{(i)}\right)^{\left(\alpha_{i}, \alpha_{i}\right)} \prod_{1 \leq i<j \leq r} \prod_{s=1}^{l_{i}} \prod_{k=1}^{l_{j}}\left(t_{s}^{(i)}-t_{k}^{(j)}\right)^{\left(\alpha_{i}, \alpha_{j}\right)},
\end{aligned}
$$

see [SV]. The function $\Phi$ is a function of variables $\boldsymbol{t}=\left(t_{j}^{(i)}\right)$, where $i=1, \ldots, r$, and $j=1, \ldots, l_{i}$, of variables $z=\left(z_{1}, \ldots, z_{n}\right)$, weights $\boldsymbol{\Lambda}$, and integers $\boldsymbol{l}$. The main variables are $\boldsymbol{t}$, the other variables will be considered as parameters.

The function $\Phi$ is symmetric with respect to permutations of variables with the same upper index.

A point $\boldsymbol{t}$ with complex coordinates is called a critical point if the following system of algebraic equations is satisfied

$$
-\sum_{s=1}^{n} \frac{\left(\Lambda_{s}, \alpha_{i}\right)}{t_{j}^{(i)}-z_{s}}+\sum_{s, s \neq i} \sum_{k=1}^{l_{s}} \frac{\left(\alpha_{s}, \alpha_{i}\right)}{t_{j}^{(i)}-t_{k}^{(s)}}+\sum_{s, s \neq j} \frac{\left(\alpha_{i}, \alpha_{i}\right)}{t_{j}^{(i)}-t_{s}^{(i)}}=0
$$

where $i=1, \ldots, r$ and $j=1, \ldots, l_{i}$. In other words, the point $\boldsymbol{t}$ is a critical point if

$$
\left(\Phi^{-1} \frac{\partial \Phi}{\partial t_{j}^{(i)}}\right)(\boldsymbol{t})=0, \quad i=1, \ldots, r, \quad j=1, \ldots, l_{i} .
$$

Note that the product of symmetric groups $S_{\boldsymbol{l}}=S_{l_{1}} \times \cdots \times S_{l_{r}}$ acts on the critical set of the master function permuting the coordinates with the same upper index. All orbits have the same cardinality $l_{1} ! \cdots l_{r}$ ! . We do not make distinction between critical points in the same orbit.

2.3. Polynomials representing critical points. Let $\boldsymbol{t}$ be a critical point of the master function $\Phi=\Phi\left(\boldsymbol{t} ; \Lambda_{\infty}\right)$. Introduce an $r$-tuple of polynomials $\boldsymbol{y}=\left(y_{1}(x), \ldots, y_{r}(x)\right)$,

$$
y_{i}(x)=\prod_{j=1}^{l_{i}}\left(x-t_{j}^{(i)}\right)
$$

Each polynomial is considered up to multiplication by a non-zero number. The tuple defines a point in the direct product $\boldsymbol{P}(\mathbb{C}[x])^{r}$ of $r$ copies of the projective space associated with the vector space of polynomials in $x$. We say that the tuple $\boldsymbol{y}$ represents the critical point.

It is convenient to think that the tuple $(1, \ldots, 1)$ of constant polynomials represents in $\boldsymbol{P}(\mathbb{C}[x])^{r}$ the critical point of the master function with no variables. This corresponds to the case when $\boldsymbol{l}=(0, \ldots, 0)$ and $\Lambda_{\infty}=\sum_{s=1}^{n} \Lambda_{s}$. 
Introduce polynomials

$$
T_{i}(x)=\prod_{s=1}^{n}\left(x-z_{s}\right)^{\left\langle\Lambda_{s}, \alpha_{i}^{\vee}\right\rangle}, \quad i=1, \ldots, r .
$$

We say that a given tuple $\boldsymbol{y}$ is generic with respect to weights $\Lambda_{1}, \ldots, \Lambda_{n}$ and points $z_{1}, \ldots, z_{n}$ if

- each polynomial $y_{i}(x)$ has no multiple roots;

- all roots of $y_{i}(x)$ are different from roots of the polynomial $T_{i}$;

- any two polynomials $y_{i}(x), y_{j}(x)$ have no common roots if $i \neq j$ and $a_{i, j} \neq 0$.

A tuple is generic if it represents a critical point.

Let $W(f, g)=f^{\prime} g-f g^{\prime}$ be the Wronskian of functions $f, g$ of $x$.

A tuple $\boldsymbol{y}$ is called fertile, if for every $i=1, \ldots, r$ there exists a polynomial $\tilde{y}_{i}$ satisfying the equation

$$
W\left(y_{i}, \tilde{y}_{i}\right)=T_{i} \prod_{j, j \neq i} y_{j}^{-\left\langle\alpha_{j}, \alpha_{i}^{\vee}\right\rangle}=T_{i} \prod_{j, j \neq i} y_{j}^{-a_{i, j}} .
$$

The polynomial $\tilde{y}_{i}$ considered up to multiplication by a non-zero number has the form

$$
\tilde{y}_{i}(x)=c_{1} y_{i}(x) \int T_{i}(x) \prod_{j=1}^{r} y_{j}(x)^{-a_{i, j}} d x+c_{2} y_{i}(x),
$$

where $c_{1}, c_{2}$ are complex numbers, $c_{1} \neq 0$.

If $\boldsymbol{y}$ is fertile and $i \in\{1, \ldots, r\}$, then the tuple

$$
\boldsymbol{y}^{[i]}=\left(y_{1}, \ldots, \tilde{y}_{i}, \ldots, y_{r}\right) \quad \in \quad \boldsymbol{P}(\mathbb{C}[x])^{r}
$$

is called the immediate descendant of $\boldsymbol{y}$ in the $i$-th direction.

Theorem 2.1 ( $\mathrm{MV1}]$ ).

(i) A generic tuple $\boldsymbol{y}=\left(y_{1}, \ldots, y_{r}\right)$, with deg $y_{j}=l_{j}$, represents a critical point of the master function $\Phi\left(\boldsymbol{t} ; \Lambda_{\infty}\right)$, with $\Lambda_{\infty}=\sum_{s=1}^{n} \Lambda_{s}-\sum_{j=1}^{r} l_{j} \alpha_{j}$, if and only if it is fertile.

(ii) If $\boldsymbol{y}$ represents a critical point, $i \in\{1, \ldots, r\}$, and $\boldsymbol{y}^{[i]}$ is an immediate descendant of $\boldsymbol{y}$, then $\boldsymbol{y}^{[i]}$ is fertile.

Let $\boldsymbol{y}$ represent a critical point of $\Phi\left(\boldsymbol{t} ; \Lambda_{\infty}\right)$. Let $i \in\{1, \ldots, r\}$ and let $\boldsymbol{y}^{[i]}$ be an immediate descendant of $\boldsymbol{y}$ in the $i$-th direction. Denote $\tilde{l}_{i}=\operatorname{deg} \tilde{y}_{i}$ and $\Lambda_{\infty}^{[i]}=\sum_{s=1}^{n} \Lambda_{s}-$ $\tilde{l}_{i} \alpha_{i}-\sum_{j=1, j \neq i}^{r} l_{j} \alpha_{j}$. Assume that $\boldsymbol{y}^{[i]}$ is generic, then $\boldsymbol{y}^{[i]}$ represents a critical point of the master function $\Phi\left(\boldsymbol{t} ; \Lambda_{\infty}^{[i]}\right)$. If $\tilde{l}_{i} \neq l_{i}$, then

$$
\Lambda_{\infty}^{[i]}=s_{i} \cdot \Lambda_{\infty},
$$

where $s_{i}$ is the shifted action of the $i$-th reflection of the Weyl group. 
2.4. Simple reproduction procedure. Let $\boldsymbol{y}$ represent a critical point of $\Phi\left(\boldsymbol{t}, \Lambda_{\infty}\right)$. The tuples $\boldsymbol{y}^{[i]}=\left(y_{1}, \ldots, \tilde{y}_{i}, \ldots, y_{r}\right) \in \boldsymbol{P}(\mathbb{C}[x])^{r}$, where $\tilde{y}_{i}$ is given by (6]) and $c_{1}, c_{2}$ are arbitrary numbers, not both equal to zero, form a one-parameter family. The parameter space of the family is identified with the projective line $\boldsymbol{P}^{1}$ with projective coordinates $\left(c_{1}: c_{2}\right)$. We have a map

$$
Y_{\boldsymbol{y}, i}: \boldsymbol{P}^{1} \rightarrow \boldsymbol{P}(\mathbb{C}[x])^{r}
$$

which sends a point $c=\left(c_{1}: c_{2}\right)$ to the corresponding tuple $\boldsymbol{y}^{[i]}$. Almost all tuples $\boldsymbol{y}^{[i]}$ are generic. The exceptions form a finite set in $\boldsymbol{P}^{1}$.

Thus, starting with a tuple $\boldsymbol{y}$, representing a critical point of the master function $\Phi\left(\boldsymbol{t} ; \Lambda_{\infty}\right)$, and an index $i \in\{1, \ldots, r\}$, we construct a family $Y_{\boldsymbol{y}, i}: \boldsymbol{P}^{1} \rightarrow \boldsymbol{P}(\mathbb{C}[x])^{r}$ of fertile tuples. For almost all $c \in \boldsymbol{P}^{1}$ (with finitely many exceptions only), the tuple $Y_{\boldsymbol{y}, i}(c)$ represents a critical point of the master function associated with integral dominant weights $\Lambda_{1}, \ldots, \Lambda_{n}$, points $z_{1}, \ldots, z_{n}$, and a suitable weight at infinity.

We call this construction the simple reproduction procedure in the $i$-th direction.

2.5. General reproduction procedure. Assume that a tuple $\boldsymbol{y} \in \boldsymbol{P}(\mathbb{C}[x])^{r}$ represents a critical point of the master function $\Phi\left(\boldsymbol{t} ; \Lambda_{\infty}\right)$.

Let $\boldsymbol{i}=\left[i_{1}, i_{2}, \ldots, i_{k}\right], i_{j} \in\{1, \ldots, r\}$, be a sequence of natural numbers. We define a $k$-parameter family of fertile tuples

$$
Y_{\boldsymbol{y}, \boldsymbol{i}}:\left(\boldsymbol{P}^{1}\right)^{k} \rightarrow \boldsymbol{P}(\mathbb{C}[x])^{r}
$$

by induction on $k$, starting at $\boldsymbol{y}$ and successively applying the simple reproduction procedure in directions $i_{1}, \ldots, i_{k}$. The image of this map is denoted $P_{\boldsymbol{y}, \boldsymbol{i}}$.

For a given $\boldsymbol{i}=\left[i_{1}, \ldots, i_{k}\right]$, almost all tuples $Y_{\boldsymbol{y}, \boldsymbol{i}}(\boldsymbol{c})$ represent critical points of master functions associated to weights $\Lambda_{1}, \ldots, \Lambda_{n}$, points $z_{1}, \ldots, z_{n}$, and suitable weights at infinity. Exceptional values of $c \in\left(\boldsymbol{P}^{1}\right)^{k}$ are contained in a proper algebraic subset.

It is easy to see that if $\boldsymbol{i}^{\prime}=\left[i_{1}^{\prime}, i_{2}^{\prime}, \ldots, i_{k^{\prime}}^{\prime}\right], i_{j} \in\{1, \ldots, r\}$, is a sequence of natural numbers, and the sequence $\boldsymbol{i}^{\prime}$ is contained in the sequence $\boldsymbol{i}$ as an ordered subset, then $P_{\boldsymbol{y}, i^{\prime}}$ is a subset of $P_{\boldsymbol{y}, \boldsymbol{i}}$.

The union

$$
P_{\boldsymbol{y}}=\cup_{\boldsymbol{i}} P_{\boldsymbol{y}, \boldsymbol{i}} \subset \boldsymbol{P}(\mathbb{C}[x])^{r},
$$

where the summation is over all of sequences $\boldsymbol{i}$, is called the population of critical points associated with the Kac-Moody algebra $\mathfrak{g}$, integral dominant weights $\Lambda_{1}, \ldots, \Lambda_{n}$, points $z_{1}, \ldots, z_{n}$, and originated at $y$.

If two populations intersect, then they coincide.

If the Weyl group is finite, then all tuples of a population consist of polynomials of bounded degree. Thus, if the Weyl group of $\mathfrak{g}$ is finite, then a population is a projective irreducible variety.

Every population $P$ has a tuple $\boldsymbol{y}=\left(y_{1}, \ldots, y_{r}\right)$, deg $y_{i}=l_{i}$, such that the weight $\Lambda_{\infty}=\sum_{s=1}^{n} \Lambda_{s}-\sum_{i=1}^{r} l_{i} \alpha_{i}$ is dominant integral, see [MV1]. 
Conjecture 2.1 (MV1]). Every population, associated with a Kac-Moody algebra $\mathfrak{g}$, dominant integral weights $\Lambda_{1}, \ldots, \Lambda_{n}$, points $z_{1}, \ldots, z_{n}$, is an algebraic variety isomorphic to the flag variety associated to the Kac-Moody algebra ${ }^{t} \mathfrak{g}$ which is Langlands dual to $\mathfrak{g}$. Moreover, the parts of the family corresponding to tuples of polynomials with fixed degrees are isomorphic to Bruhat cells of the flag variety.

The conjecture is proved for the Lie algebras with root systems of types $A_{r}, B_{r}, C_{r}, G_{2}$ in [MV1, BM]. In Theorems 4.2 and 5.1 we prove this conjecture for any simple Lie algebra.

2.6. Diagonal sequences of polynomials associated with a critical point and a sequence of indices. We introduce notions which will be used in Chapter [6 to construct solutions of differential equations.

Lemma 2.2. Assume that a tuple $\boldsymbol{y}$ of non-zero polynomials represents a critical point of the master function $\Phi\left(\boldsymbol{t} ; \Lambda_{\infty}\right)$. Let $\boldsymbol{i}=\left[i_{1}, i_{2}, \ldots, i_{k}\right], i_{j} \in\{1, \ldots, r\}$, be a sequence of natural numbers. Then there exist tuples $\boldsymbol{y}^{\left[i_{1}\right]}=\left(y_{1}^{\left[i_{1}\right]}, \ldots, y_{r}^{\left[i_{1}\right]}\right), \boldsymbol{y}^{\left[i_{1}, i_{2}\right]}=$ $\left(y_{1}^{\left[i_{1}, i_{2}\right]}, \ldots, y_{r}^{\left[i_{1}, i_{2}\right]}\right), \ldots, \boldsymbol{y}^{\left[i_{1}, i_{2}, \ldots, i_{k}\right]}=\left(y_{1}^{\left[i_{1}, i_{2}, \ldots, i_{k}\right]}, \ldots, y_{r}^{\left[i_{1}, i_{2}, \ldots, i_{k}\right]}\right)$ in $\boldsymbol{P}(\mathbb{C}[x])^{r}$ such that

$$
W\left(y_{i_{1}}, y_{i_{1}}^{\left[i_{1}\right]}\right)=T_{i_{1}} \prod_{j, j \neq i_{1}} y_{j}^{-a_{i_{1}, j}}
$$

and $y_{j}^{\left[i_{1}\right]}=y_{j}$ for $j \neq i_{1}$;

(ii) for $l=2, \ldots, k$, we have

$$
\begin{aligned}
& W\left(y_{i_{l}}^{\left[i_{1}, \ldots, i_{l-1}\right]}, y_{i_{l}}^{\left[i_{1}, \ldots, i_{l}\right]}\right)=T_{i_{l}} \prod_{j, j \neq i_{l}}\left(y_{j}^{\left[i_{1}, \ldots, i_{l-1}\right]}\right)^{-a_{i_{l}, j}} \\
& \text { and } y_{j}^{\left[i_{1}, \ldots, i_{l}\right]}=y_{j}^{\left[i_{1}, \ldots, i_{l-1}\right]} \text { for } j \neq i_{l} .
\end{aligned}
$$

The tuples $\boldsymbol{y}^{\left[i_{1}\right]}, \boldsymbol{y}^{\left[i_{1}, i_{2}\right]}, \ldots, \boldsymbol{y}^{\left[i_{1}, i_{2}, \ldots, i_{k}\right]}$ belong to the population $P_{\boldsymbol{y}}$. The tuple $\boldsymbol{y}^{\left[i_{1}\right]}$ is obtained from $\boldsymbol{y}$ by the $i_{1}$-th simple generation procedure and for $l=2, \ldots, k$, the tuple $\boldsymbol{y}^{\left[i_{1}, \ldots, i_{l}\right]}$ is obtained from $\boldsymbol{y}^{\left[i_{1}, \ldots, i_{l-1}\right]}$ by the $i_{l}$-th simple generation procedure.

The sequence of tuples $\boldsymbol{y}^{\left[i_{1}\right]}, \boldsymbol{y}^{\left[i_{1}, i_{2}\right]}, \ldots, \boldsymbol{y}^{\left[i_{1}, i_{2}, \ldots, i_{k}\right]}$ satisfying Lemma [2.2 will be called associated with the critical point $\boldsymbol{y}$ and the sequence of indices $\boldsymbol{i}$. The sequence of polynomials $y_{i_{1}}^{\left[i_{1}\right]}, y_{i_{2}}^{\left[i_{1}, i_{2}\right]}, \ldots, y_{i_{k}}^{\left[i_{1}, i_{2}, \ldots, i_{k}\right]}$ will be called the diagonal sequence of polynomials associated with the critical point $\boldsymbol{y}$ and the sequence of indices $\boldsymbol{i}$. For a given $\boldsymbol{y}$ the diagonal sequence of polynomials determine the sequence of tuples $\boldsymbol{y}^{\left[i_{1}\right]}, \boldsymbol{y}^{\left[i_{1}, i_{2}\right]}, \ldots$, $\boldsymbol{y}^{\left[i_{1}, i_{2}, \ldots, i_{k}\right]}$ uniquely.

There are many diagonal sequences of polynomials associated with a given critical point and a given sequence of indices. 


\section{OpERS}

Let $\mathfrak{g}=\mathfrak{g}(A)$ be a Kac-Moody algebra with simple roots $\alpha_{1}, \ldots, \alpha_{r}$ and simple coroots $\alpha_{1}^{\vee}, \ldots, \alpha_{r}^{\vee}$. Let ${ }^{t} \mathfrak{g}=\mathfrak{g}\left({ }^{t} A\right)$ be the Langlands dual algebra with Chevalley generators ${ }^{t} \mathfrak{h}, E_{1}, \ldots, E_{r}, F_{1}, \ldots, F_{r}$, simple roots ${ }^{t} \alpha_{1}, \ldots,{ }^{t} \alpha_{r}$ and simple coroots ${ }^{t} \alpha_{1}^{\vee}, \ldots,{ }^{t} \alpha_{r}^{\vee}$. Set $H_{1}={ }^{t} \alpha_{1}^{\vee}, \ldots, H_{r}={ }^{t} \alpha_{r}^{\vee}$ and

$$
I=F_{1}+\cdots+F_{r}, \quad \partial=d / d x .
$$

$A^{t} \mathfrak{g}$-oper is a differential operator of the form

$$
D=\partial+I+V+W
$$

with $V \in M\left({ }^{t} \mathfrak{h}\right)$ and $W \in \bar{M}\left({ }^{t} \mathfrak{n}_{+}\right)$. A Miura ${ }^{t} \mathfrak{g}$-oper is a differential operator of the form

$$
D=\partial+I+V
$$

with $V \in M\left({ }^{t} \mathfrak{h}\right)$.

The differential operators of that type were considered by V. Drinfeld and V. Sokolov in their study of the KdV type equations [DS]. On opers and Miura opers see [BD, FFR, F1, F2, MV1, BM, MV4.

For $u \in \bar{M}\left({ }^{t} \mathfrak{n}_{+}\right)$and a ${ }^{t} \mathfrak{g}$-oper $D$, the differential operator

$$
e^{\operatorname{ad} u} \cdot D=D+[u, D]+\frac{1}{2}[u,[u, D]]+\ldots
$$

is a ${ }^{t} \mathfrak{g}$-oper. The opers $D$ and $e^{\text {ad } u} \cdot D$ are called gauge equivalent.

Let $X$ be a ${ }^{t} \mathfrak{g}$-module with locally finite action of ${ }^{t} \mathfrak{n}_{+}$. Let $D$ be a ${ }^{t} \mathfrak{g}$-oper and $u \in \bar{M}\left({ }^{t} \mathfrak{n}_{+}\right)$. Then $D, e^{\text {ad } u} \cdot D, e^{ \pm u}$ determine linear operators on $M(X)$. Moreover, we have

$$
e^{\operatorname{ad} u} \cdot D=e^{u} D e^{-u}
$$

Lemma 3.1. Let $D=\partial+I+V$ be a Miura ${ }^{t} \mathfrak{g}$-oper. Let $g \in M(\mathbb{C})$ and $i \in\{1, \ldots, r\}$. Then

$$
e^{a d\left(g E_{i}\right)} \cdot D=\partial+I+\left(V+g H_{i}\right)-\left(g^{\prime}+\left\langle{ }^{t} \alpha_{i}, V\right\rangle g+g^{2}\right) E_{i} .
$$

The proof is straightforward.

Corollary 3.2. Let $D=\partial+I+V$ be a Miura ${ }^{t} \mathfrak{g}$-oper. Then the ${ }^{t} \mathfrak{g}$-oper $e^{\text {ad }\left(g E_{i}\right)} \cdot D$ is a Miura oper if and only if the scalar rational function $g$ satisfies the Ricatti equation

$$
g^{\prime}+\left\langle{ }^{t} \alpha_{i}, V\right\rangle g+g^{2}=0
$$

We say that the Miura ${ }^{t} \mathfrak{g}$-oper $D$ is deformable in the $i$-th direction if equation (8) has a non-zero solution which is a rational function.

Fix a collection of dominant integral weights $\boldsymbol{\Lambda}=\left(\Lambda_{1}, \ldots, \Lambda_{n}\right)$ of the Kac-Moody algebra $\mathfrak{g}$, and numbers $\boldsymbol{z}=\left\{z_{1}, \ldots, z_{n}\right\} \subset \mathbb{C}, z_{i} \neq z_{j}$ if $i \neq j$. Introduce polynomials $T_{1}(x), \ldots, T_{r}(x)$ by formulas (41). 
Let $\boldsymbol{y}=\left(y_{1}, \ldots, y_{r}\right)$ be a tuple of non-zero polynomials. We say that a Miura ${ }^{t} \mathfrak{g}$-oper $D=\partial+I+V$ is associated with weights $\boldsymbol{\Lambda}$, numbers $\boldsymbol{z}$, and the tuple $\boldsymbol{y}=\left(y_{1}, \ldots, y_{r}\right)$, if for every $i \in\{1, \ldots, r\}$ we have

$$
\left\langle{ }^{t} \alpha_{i}, V\right\rangle=-\log ^{\prime}\left(T_{i} \prod_{j=1}^{r} y_{j}^{-\left\langle\alpha_{j}, \alpha_{i}^{\vee}\right\rangle}\right)=-\log ^{\prime}\left(T_{i} \prod_{j=1}^{r} y_{j}^{-a_{i, j}}\right),
$$

cf. (5).

If a Miura oper $D$ is associated with weights $\boldsymbol{\Lambda}$, numbers $\boldsymbol{z}$, and a generic tuple $\boldsymbol{y}=$ $\left(y_{1}, \ldots, y_{r}\right) \in \boldsymbol{P}(\mathbb{C}[x])^{r}$, then the tuple $\boldsymbol{y}$ is determined uniquely. Indeed, the residues of the rational function $\left\langle{ }^{t} \alpha_{i}, V\right\rangle$ are positive exactly at the roots of the polynomial $y_{i}$ and the residues are equal to the multiplicities of roots of $y_{i}$ multiplied by two.

If $\mathfrak{g}$ is a simple Lie algebra, then $D$ determines $\boldsymbol{y}$ uniquely even if $\boldsymbol{y}$ is not generic. That fact follows from the invertibility of the Cartan matrix of $\mathfrak{g}$.

Theorem 3.1. Let the Miura ${ }^{t} \mathfrak{g}$-oper $D=\partial+I+V$ be associated with weights $\boldsymbol{\Lambda}$, numbers $\boldsymbol{z}$, and the tuple $\boldsymbol{y}=\left(y_{1}, \ldots, y_{r}\right)$. Let $i \in\{1, \ldots, r\}$. Then $D$ is deformable in the $i$-th direction if and only if there exists a polynomial $\tilde{y}_{i}$ satisfying (5). Moreover, in that case any non-zero rational solution $g$ of the Ricatti equation (8) has the form $g=\log ^{\prime}\left(\tilde{y}_{i} / y_{i}\right)$ where $\tilde{y}_{i}$ is a solution of (5). If $g=\log ^{\prime}\left(\tilde{y}_{i} / y_{i}\right)$, then the Miura ${ }^{t} \mathfrak{g}$-oper

$$
e^{a d\left(g E_{i}\right)} \cdot D=\partial+I+\left(V+g H_{i}\right)
$$

is associated with weights $\boldsymbol{\Lambda}$, numbers $\boldsymbol{z}$, and the tuple $\boldsymbol{y}^{[i]}=\left(y_{1}, \ldots, \tilde{y}_{i}, \ldots, y_{r}\right)$, where the tuple $\boldsymbol{y}^{[i]}$ is called in Section [2.3 an immediate descendant of $\boldsymbol{y}$ in the $i$-th direction, see (7).

Proof. Write (8) as

$$
g^{\prime} / g+g=\log ^{\prime}\left(T_{i} \prod_{j=1}^{r} y_{j}^{-a_{i, j}}\right)
$$

If $g$ is a rational function, then $g \rightarrow 0$ as $x \rightarrow \infty$ and all poles of $g$ are simple. Moreover, the residue of $g$ at any point is an integer. Hence $g=c^{\prime} / c$ for a suitable rational function c. Then

$$
c=\int T_{i}(x) \prod_{j=1}^{r} y_{j}(x)^{-a_{i, j}} d x
$$

and equation (5) has a polynomial solution $\tilde{y}_{i}=-c y_{i}$. Conversely if equation (5) has a polynomial solution $\tilde{y}_{i}$, then the function $c$ in (12) is rational. Then $g=c^{\prime} / c$ is a rational solution of equation (8).

Let $g=\log ^{\prime}(c)=\log ^{\prime}\left(\tilde{y}_{i} / y_{i}\right)$, where $\tilde{y}_{i}$ is a solution of (5). Then

$$
e^{\operatorname{ad}\left(g E_{i}\right)} \cdot D=\partial+I+\left(V+\log ^{\prime}\left(\tilde{y}_{i} / y_{i}\right) H_{i}\right)
$$


and

$$
\left\langle{ }^{t} \alpha_{k}, V\right\rangle+\log ^{\prime}\left(\tilde{y}_{i} / y_{i}\right)\left\langle{ }^{t} \alpha_{k},{ }^{t} \alpha_{i}^{\vee}\right\rangle=-\log ^{\prime}\left(T_{k} \tilde{y}_{i}^{-\left\langle\alpha_{i}, \alpha_{k}^{\vee}\right\rangle} \prod_{j=1, j \neq i}^{r} y_{j}^{-\left\langle\alpha_{j}, \alpha_{k}^{\vee}\right\rangle}\right) .
$$

Note that if equation (8) has one non-zero rational solution $g=c^{\prime} / c$ with rational $c$, then other non-zero (rational) solutions have the form $g=c^{\prime} /(c+$ const $)$.

Corollary 3.3. Let the Miura ${ }^{t} \mathfrak{g}$-oper $D=\partial+I+V$ be associated with weights $\boldsymbol{\Lambda}$, numbers $\boldsymbol{z}$, and the tuple $\boldsymbol{y}=\left(y_{1}, \ldots, y_{r}\right)$. Then $D$ is deformable in all directions from 1 to $r$ if and only if the tuple $\boldsymbol{y}$ is fertile.

Corollary 3.4. Let the Miura ${ }^{t} \mathfrak{g}$-oper $D=\partial+I+V$ be associated with weights $\boldsymbol{\Lambda}$, numbers $\boldsymbol{z}$, and the tuple $\boldsymbol{y}=\left(y_{1}, \ldots, y_{r}\right)$. Let the tuple $\boldsymbol{y}=\left(y_{1}, \ldots, y_{r}\right)$ be generic in the sense of Section 2.3. Then $D$ is deformable in all directions from 1 to $r$ if and only if the tuple $\boldsymbol{y}$ represents a critical point of the master function (1) associated with parameters $\boldsymbol{z}, \boldsymbol{\Lambda}, \Lambda_{\infty}$.

Let the Miura ${ }^{t} \mathfrak{g}$-oper $D=\partial+I+V$ be associated with weights $\boldsymbol{\Lambda}$, numbers $\boldsymbol{z}$, and the tuple $\boldsymbol{y}=\left(y_{1}, \ldots, y_{r}\right)$. Let the tuple $\boldsymbol{y}=\left(y_{1}, \ldots, y_{r}\right)$ represent a critical point of the master function (11) associated with parameters $\boldsymbol{z}, \boldsymbol{\Lambda}, \Lambda_{\infty}$. Let $\mathrm{Om}_{\mathrm{D}}{ }^{0}$ be the variety of all Miura opers which can be obtained from $D$ by a sequence of deformations in directions $i_{1}, \ldots, i_{N}$ where $N$ is any positive integer and all $i_{j}$ lie in $\{1, \ldots, r\}$.

Corollary 3.5. For a simple Lie algebra $\mathfrak{g}$ the variety $\mathrm{Om}_{\mathrm{D}}{ }^{0}$ is isomorphic to the population of critical points originated at $\boldsymbol{y}$.

\section{MiURA OPERS AND FLAG VARIETIES}

In this section we assume that $\mathfrak{g}$ and ${ }^{t} \mathfrak{g}$ are simple Lie algebras although most of considerations can be extended to Kac-Moody algebras.

Let ${ }^{t} G$ be the complex simply connected Lie group with Lie algebra ${ }^{t} \mathfrak{g}$. Let ${ }^{t} B_{ \pm},{ }^{t} N_{ \pm}$, ${ }^{t} H$ be the subgroups with Lie algebras ${ }^{t} \mathfrak{b}_{ \pm},{ }^{t} \mathfrak{n}_{ \pm},{ }^{t} \mathfrak{h}$, respectively.

4.1. Triviality of the monodromy. Let $D=\partial+I+V$ be a Miura ${ }^{t} \mathfrak{g}$-oper. Let $\mathbb{P}^{1}$ be the complex projective line. Consider $D$ as a ${ }^{t} G$-connection $\nabla_{D}$ on the trivial principal ${ }^{t} G$-bundle $p:{ }^{t} G \times \mathbb{P}^{1} \rightarrow \mathbb{P}^{1}$. The connection has singularities at the set Sing $\subset \mathbb{P}^{1}$ where the function $V$ has poles. Choose a regular point $x_{0} \in \mathbb{P}^{1}-\infty$ of the connection. Parallel translations with respect to the connection define the monodromy representation $\pi\left(\mathbb{P}^{1}-\mathrm{Sing}\right) \rightarrow{ }^{t} G$. Its image is called the monodromy group.

Theorem 4.1. Assume that the Miura ${ }^{t} \mathfrak{g}$-oper $D$ is associated with weights $\boldsymbol{\Lambda}$, numbers $\boldsymbol{z}$, and a tuple $\boldsymbol{y}=\left(y_{1}, \ldots, y_{r}\right)$ as in Section $\mathbf{3}$. Assume that the tuple $\boldsymbol{y}=\left(y_{1}, \ldots, y_{r}\right)$ is generic in the sense of Section [2.3. Assume that the Miura oper D is deformable in all directions from 1 to $r$. Then the monodromy group of $\nabla_{D}$ belongs to the center of ${ }^{t} G$. 
Proof. It is known that the intersection of all of the Borel subgroups in ${ }^{t} G$ is the center of ${ }^{t} G$, see $\left[\mathrm{B},[\mathrm{H}]\right.$. We show that the monodromy of $\nabla_{D}$ lies in the intersection of all of the Borel subgroups.

Let id $\in{ }^{t} G$ be the identity element. Let $\bar{Y}(x) \in{ }^{t} G$ be the (possibly multi-valued) solution of the equation $D Y=(\partial+I+V) Y=0$ such that $Y\left(x_{0}\right)=$ id. Since $D$ is a Miura oper we have for any regular $x$ the equality of sets $\bar{Y}(x){ }^{t} B_{-}={ }^{t} B_{-}$. Hence if $m \in{ }^{t} G$ is an element of the monodromy group of $\nabla_{D}$, then $m{ }^{t} B_{-}={ }^{t} B_{-}$and hence $m \in{ }^{t} B_{-}$.

Let $i \in\{1, \ldots, r\}$ and let $g_{i} \in M(\mathbb{C})$ be a solution of the Ricatti equation (8) . Assume that $g_{i}$ is regular at $x_{0}$. Then the ${ }^{t} G$-valued function

$$
e^{g_{i}(x) E_{i}} \bar{Y}(x) e^{-g_{i}\left(x_{0}\right) E_{i}}
$$

is the solution of the equation $\left(e^{\operatorname{ad}\left(g_{i}(x) E_{i}\right)} \cdot D\right) Y=0$ such that $Y\left(x_{0}\right)=$ id. Since $e^{\operatorname{ad}\left(g_{i}(x) E_{i}\right)} \cdot D$ is a Miura oper we have an equality of sets $Y_{g_{i}}^{[i]}(x){ }^{t} B_{-}={ }^{t} B_{-}$for any $x$ at which $e^{\text {ad }\left(g_{i}(x) E_{i}\right)} \cdot D$ is regular. Hence for any element $m$ of the monodromy group of $\nabla_{D}$ we have

$$
e^{g_{i}\left(x_{0}\right) E_{i}} m e^{-g_{i}\left(x_{0}\right) E_{i} t} B_{-} \subset{ }^{t} B_{-}
$$

or $m \in e^{-g_{i}\left(x_{0}\right) E_{i} t} B_{-} e^{g_{i}\left(x_{0}\right) E_{i}}$.

Now consider the Miura oper

$$
e^{\operatorname{ad}\left(g_{i}(x) E_{i}\right)} \cdot D=\partial+I+V_{i ; g_{i}}
$$

where $V_{i ; g_{i}}$ is the ${ }^{t} \mathfrak{h}$-part of $e^{\operatorname{ad}\left(g_{i}(x) E_{i}\right)} \cdot D$. Let $j \in\{1, \ldots, r\}$ and let $g_{i, j ; g_{i}} \in M(\mathbb{C})$ be a solution of the $j$-th Ricatti equation

$$
g^{\prime}+\left\langle{ }^{t} \alpha_{i}, V_{i ; g_{i}}\right\rangle g+g^{2}=0
$$

associated with the Miura oper $e^{\text {ad }\left(g_{i}(x) E_{i}\right)} \cdot D$. Assume that $g_{i, j ; g_{i}}$ is regular at $x_{0}$. Then the ${ }^{t} G$-valued function

$$
e^{g_{i, j ; g_{i}}(x) E_{j}} e^{g_{i}(x) E_{i}} \bar{Y}(x) e^{-g_{i}\left(x_{0}\right) E_{i}} e^{-g_{i, j ; g_{i}}\left(x_{0}\right) E_{j}}
$$

is the solution of the equation $\left(e^{\operatorname{ad}\left(g_{i, j ; g_{i}}(x) E_{j}\right)} e^{\operatorname{ad}\left(g_{i}(x) E_{i}\right)} \cdot D\right) Y=0$ such that $Y\left(x_{0}\right)=$ id. Repeating the previous argument we conclude that any element $m$ of the monodromy group of $\nabla_{D}$ lies in the Borel subgroup

$$
e^{-g_{i}\left(x_{0}\right) E_{i}} e^{-g_{i, j ; g_{i}}\left(x_{0}\right) E_{j} t} B_{-} e^{g_{i, j ; g_{i}}\left(x_{0}\right) E_{j}} e^{g_{i}\left(x_{0}\right) E_{i}} .
$$

Every $u \in{ }^{t} N_{+}$is a product of elements of the form $e^{c_{i} E_{i}}$ for $i \in\{1, \ldots, r\}$ and $c_{i} \in \mathbb{C}$. Every $c_{i}$ can be taken as the initial condition for a solution of the suitable $i$-th Ricatti equation. Therefore the iteration of the previous reason shows that every element of the monodromy group of $\nabla_{D}$ lies in every Borel subgroup of the form $u^{-1}\left({ }^{t} B_{-}\right) u, u \in{ }^{t} N_{+}$. The Borel subgroups in ${ }^{t} G$ of the form $u^{-1}\left({ }^{t} B_{-}\right) u, u \in{ }^{t} N_{+}$, form an open dense subset in the flag variety of all of the Borel subgroups. Hence the monodromy lies in the intersection of all of the Borel subgroups. 
4.2. Gauge equivalent Miura opers. As in Section 4.1, let $D$ be the Miura ${ }^{t} G$-oper associated with weights $\boldsymbol{\Lambda}$, numbers $\boldsymbol{z}$, and a tuple $\boldsymbol{y}=\left(y_{1}, \ldots, y_{r}\right)$. We assume that the tuple $\boldsymbol{y}=\left(y_{1}, \ldots, y_{r}\right)$ is generic in the sense of Section 2.3 and the Miura oper $D$ is deformable in all directions from 1 to $r$.

Consider the variety $\mathrm{Om}_{\mathrm{D}}$ of all Miura opers gauge equivalent to $D$. If $D^{\prime} \in \mathrm{Om}_{\mathrm{D}}$, then there exists a rational $N_{+}$-valued function $v$ on $\mathbb{P}^{1}$ such that $D^{\prime}=v D v^{-1}$. In that case we denote $D^{\prime}$ by $D^{v}$.

Let $\mathrm{Om}_{\mathrm{D}}{ }^{0} \subseteq \mathrm{Om}_{\mathrm{D}}$ be the subvariety of all Miura opers which can be obtained from $D$ by a sequence of deformations in directions $i_{1}, \ldots, i_{N}$ where $N$ is a non-negative integer and all $i_{j}$ lie in $\{1, \ldots, r\}$. By Corollary 3.5 the subvariety $\mathrm{Om}_{\mathrm{D}}{ }^{0}$ is isomorphic to the population of critical points originated at $\boldsymbol{y}$.

The connection $\nabla_{D}$ is regular at $x_{0} \in \mathbb{P}^{1}$ if $x_{0}$ does not lie in $\left\{z_{1}, \ldots, z_{n}, \infty\right\}$ and $x_{0}$ is not a root of some of polynomials $y_{1}, \ldots, y_{r}$.

Consider the trivial bundle $p^{\prime}:\left({ }^{t} G /{ }^{t} B_{-}\right) \times \mathbb{P}^{1} \rightarrow \mathbb{P}^{\mathbf{1}}$ associated with the bundle $p$. The fiber of $p^{\prime}$ is the flag variety ${ }^{t} G /{ }^{t} B_{-}$. The connection $\nabla_{D}$ induces a connection $\nabla_{D}^{\prime}$ on $p^{\prime}$. The monodromy of $\nabla_{D}^{\prime}$ is trivial by Theorem 4.1. Thus the variety $\Gamma$ of global horizontal sections of $\nabla_{D}^{\prime}$ is identified with the fiber $\left(p^{\prime}\right)^{-1}\left(x_{0}\right)$ over any $x_{0}$ which is a regular point of the connection. Thus $\Gamma$ is isomorphic to ${ }^{t} G /{ }^{t} B_{-}$.

Any ${ }^{t} G$-valued rational function $v$ defines a section

$$
S_{v}: x \mapsto v(x)^{-1} B_{-} \times x
$$

of $p^{\prime}$ over the set of regular points of $v$. The section $S_{v}$ is also well defined over the poles of $v$ since ${ }^{t} G /{ }^{t} B_{-}$is a projective variety.

If $D^{v} \in \mathrm{Om}_{\mathrm{D}}$, then the section $S_{v}$ is horizontal with respect to $\nabla_{D}^{\prime}$, cf. the proof of Theorem 4.1. Thus we have a map

$$
S: \mathrm{Om}_{\mathrm{D}} \rightarrow \Gamma, \quad D^{v} \mapsto S_{v}
$$

Theorem 4.2. The map $S: \mathrm{Om}_{\mathrm{D}} \rightarrow \Gamma$ is an isomorphism and $\mathrm{Om}_{\mathrm{D}}{ }^{0}=\mathrm{Om}_{\mathrm{D}}$.

Proof. Let $D^{v_{1}}, D^{v_{2}} \in \mathrm{Om}_{\mathrm{D}}$. Assume that the images of $D^{v_{1}}$ and $D^{v_{2}}$ under the map $S$ coincide. Assume that $v_{1}, v_{2}, D$ are regular at $x_{0} \in \mathbb{P}^{1}$. The equality $S_{v_{1}}\left(x_{0}\right)=S_{v_{2}}\left(x_{0}\right)$ means that $v_{1}\left(x_{0}\right)^{-1 t} B_{-}=v_{2}\left(x_{0}\right)^{-1 t} B_{-}$. Then $v_{1}\left(x_{0}\right)=v_{2}\left(x_{0}\right)$. Hence $v_{1}=v_{2}$ and $D^{v_{1}}=D^{v_{2}}$. That proves the injectivity of $S$.

Let $x_{0}$ be a regular point of $D$ in $\mathbb{P}^{1}-\infty$. For any $u \in{ }^{t} N_{+}$there exists a rational ${ }^{t} N_{+}$-valued function $v$ such that $v\left(x_{0}\right)=u$ and $D^{v} \in \mathrm{Om}_{\mathrm{D}}$ and $D^{v}$ is obtained from $D$ by a sequence of deformations in some directions $i_{1}, \ldots, i_{N}$, see the proof of Theorem 4.1. Thus the set

$$
\operatorname{Im}\left(x_{0}\right)=\left\{S_{v}\left(x_{0}\right) \in\left({ }^{t} G /{ }^{t} B_{-}\right) \times x_{0} \mid D^{v} \in \mathrm{Om}_{\mathrm{D}}{ }^{0}\right\}
$$

contains the set $\left(\left({ }^{t} N_{+}{ }^{t} B_{-}\right) /{ }^{t} B_{-}\right) \times x_{0} \subset\left({ }^{t} G /{ }^{t} B_{-}\right) \times x_{0}$. The set $\operatorname{Im}\left(x_{0}\right)$ is the image with respect to $S$ of a population of critical points. Hence it is closed as the image of a closed variety. On the other hand the set $\left(\left({ }^{t} N_{+}{ }^{t} B_{-}\right) /{ }^{t} B_{-}\right) \times x_{0}$ is dense in $\left({ }^{t} G /{ }^{t} B_{-}\right) \times x_{0}$. 
Hence $\operatorname{Im}\left(x_{0}\right)=\left({ }^{t} G /{ }^{t} B_{-}\right) \times x_{0}$ and $S\left(\mathrm{Om}_{\mathrm{D}}{ }^{0}\right)=\Gamma$. Therefore $\mathrm{Om}_{\mathrm{D}}{ }^{0}=\mathrm{Om}_{\mathrm{D}}$ since the map $S$ is injective.

4.3. Remarks on the isomorphism. Let $\mathfrak{g}$ be a simple Lie algebra. Let $P_{\boldsymbol{y}_{0}}$ be the population of critical points originated at a tuple $\boldsymbol{y}^{0}$. We assume that the tuple $\boldsymbol{y}^{0}$ is generic in the sense of Section 2.3. Theorem 4.2 says that the population $P_{\boldsymbol{y}^{0}}$ is isomorphic to the flag variety ${ }^{t} G /{ }^{t} B_{-}$. The isomorphism is constructed in three steps. If $\boldsymbol{y}^{\prime} \in P_{\boldsymbol{y}}$ is a point of the population, then one assigns to it the associated Miura oper $D_{\boldsymbol{y}^{\prime}}$ as in Section 3, see also Lemma 6.1. By Theorem 3.1 we have $D_{\boldsymbol{y}^{\prime}}=v D_{\boldsymbol{y}^{0}} v^{-1}$ for a suitable rational function $v: \mathbb{P}^{1} \rightarrow{ }^{t} N_{+}$. To the Miura oper $D^{v}$ one assigns the section $S_{v} \in \Gamma$ by formula (13). Then one chooses a point $x_{0} \in \mathbb{C}$, regular with respect to the connection $\nabla_{D_{y^{0}}}$, and assigns to a section $S^{v}$ its value $S^{v}\left(x_{0}\right) \in\left({ }^{t} G /{ }^{t} B_{-}\right) \times x_{0}$. The resulting composition

$$
\phi_{\boldsymbol{y}^{0}, x_{0}}: P_{\boldsymbol{y}} \rightarrow{ }^{t} G /{ }^{t} B_{-}
$$

is an isomorphism according to Theorem 4.2 .

Lemma 4.1. If $x_{0}, x_{1} \in \mathbb{C}$ are points regular with respect to $\nabla_{D_{y^{0}}}$, then there exists an element $g \in{ }^{t} B_{-}$such that $\phi_{\boldsymbol{y}^{0}, x_{1}}=g \phi_{\boldsymbol{y}^{0}, x_{0}}$.

Proof. Let $Y$ be the ${ }^{t} G$-valued solution of the equation $D_{\boldsymbol{y}^{0}} Y=0$ such that $Y\left(x_{0}\right)=\mathrm{id}$. Then $Y(x) \in{ }^{t} B_{-}$for all $x$. If $D^{v} \in \mathrm{Om}_{\mathrm{D} \boldsymbol{y}^{0}}$, then $S^{v}$ is a horizontal section of $\nabla_{D_{\boldsymbol{y}^{0}}}^{\prime}$. Thus it has the form $x \mapsto\left(Y(x) u{ }^{t} B_{-}\right) \times x$ for a suitable element $u \in{ }^{t} G$. Hence $\phi_{\boldsymbol{y}^{0}, x_{0}}\left(\boldsymbol{y}^{\prime}\right)=Y\left(x_{0}\right) u^{t} B_{-}$and $\phi_{\boldsymbol{y}^{0}, x_{1}}\left(\boldsymbol{y}^{\prime}\right)=Y\left(x_{1}\right) u^{t} B_{-}$. We conclude that $\phi_{\boldsymbol{y}^{0}, x_{1}}=$ $Y\left(x_{1}\right) Y\left(x_{0}\right)^{-1} \phi_{\boldsymbol{y}^{0}, x_{0}}$.

Let $\boldsymbol{y}^{1}$ be a point of $P_{\boldsymbol{y}^{0}}$. Let $P_{\boldsymbol{y}^{1}}$ be the population originated at $\boldsymbol{y}^{1}$. We have $P_{\boldsymbol{y}^{0}}=P_{\boldsymbol{y}^{1}}$.

Lemma 4.2. Let $x_{0} \in \mathbb{C}$ be regular with respect to both connections $\nabla_{D_{y^{0}}}$ and $\nabla_{D_{y^{1}}}$. Then there exists an element $g \in{ }^{t} B_{+}$such that $\phi_{\boldsymbol{y}^{1}, x_{0}}=g \phi_{\boldsymbol{y}^{0}, x_{0}}$.

Proof. We have $D_{\boldsymbol{y}^{1}}=w D_{\boldsymbol{y}^{0}} w^{-1}$ for a suitable rational function $w: \mathbb{P}^{1} \rightarrow{ }^{t} N_{+}$. If $Y_{0}(x)$ is the ${ }^{t} G$-valued solution of the equation $D_{\boldsymbol{y}^{0}} Y=0$ such that $Y_{0}\left(x_{0}\right)=\mathrm{id}$, then $Y_{1}(x)=w(x) Y(x) w\left(x_{0}\right)^{-1}$ is the ${ }^{t} G$-valued solution of the equation $D_{\boldsymbol{y}^{1}} Y=0$ such that $Y_{1}\left(x_{0}\right)=\mathrm{id}$.

Let $\boldsymbol{y}^{\prime} \in P_{\boldsymbol{y}^{0}}$ and $D_{\boldsymbol{y}^{\prime}}=v D_{\boldsymbol{y}^{0}} v^{-1}$ for a suitable rational function $v: \mathbb{P}^{1} \rightarrow{ }^{t} N_{+}$. Then $D_{\boldsymbol{y}^{\prime}}=v w^{-1} D_{\boldsymbol{y}^{1}} w v^{-1}$. Hence $\phi_{\boldsymbol{y}^{0}, x_{0}}\left(\boldsymbol{y}^{\prime}\right)=v\left(x_{0}\right)^{-1 t} B_{-}$and $\phi_{\boldsymbol{y}^{1}, x_{0}}\left(\boldsymbol{y}^{\prime}\right)=$ $w\left(x_{0}\right) v\left(x_{0}\right)^{-1 t} B_{-}$. Therefore, $\phi_{\boldsymbol{y}^{1}, x_{0}}=w\left(x_{0}\right) \phi_{\boldsymbol{y}^{0}, x_{0}}$.

\section{Bruhat Cells}

5.1. Properties of Bruhat cells. Let $\mathfrak{g}$ be a simple Lie algebra. For an element $w$ of the Weyl group $W$, the set

$$
B_{w}={ }^{t} B_{-} w^{t} B_{-} \subset{ }^{t} G /{ }^{t} B_{-}
$$


is called the Bruhat cell associated to $w$. The Bruhat cells form a cell decomposition of the flag variety ${ }^{t} G /{ }^{t} B_{-}$.

For $w \in W$ denote $l(w)$ the length of $w$. We have $\operatorname{dim} B_{w}=l(w)$.

Let $s_{1}, \ldots, s_{r} \in W$ be the generating reflections of the Weyl group.

For $v \in{ }^{t} G /{ }^{t} B_{-}$and $i \in\{1, \ldots, r\}$ consider the rational curve

$$
\mathbb{C} \rightarrow{ }^{t} G /{ }^{t} B_{-}, \quad c \mapsto e^{c E_{i}} v .
$$

The limit of $e^{c E_{i}} v$ is well defined as $c \rightarrow \infty$, since ${ }^{t} G /{ }^{t} B_{-}$is a projective variety.

We need the following standard property of Bruhat cells.

Lemma 5.1. Let $s_{i}, w \in W$ be such that $l\left(s_{i} w\right)=l(w)+1$. Then

$$
B_{s_{i} w}=\left\{e^{c E_{i}} v \mid v \in B_{w}, c \in\left\{\mathbb{P}^{1}-0\right\}\right\} .
$$

Corollary 5.2. Let $w=s_{i_{1}} \cdots s_{i_{k}}$ be a reduced decomposition of $w \in W$. Then

$$
B_{w}=\left\{\lim _{c_{1} \rightarrow c_{1}^{0}} \ldots \lim _{c_{k} \rightarrow c_{k}^{0}} e^{c_{1} E_{i_{1}}} \ldots e^{c_{k} E_{i_{k}}{ }^{t}} B_{-} \in{ }^{t} G /{ }^{t} B_{-} \mid c_{1}^{0}, \ldots, c_{k}^{0} \in\left\{\mathbb{P}^{1}-0\right\}\right\} .
$$

Introduce the map

$$
f_{i_{1}, \ldots, i_{k}}:(\mathbb{C}-0)^{k} \rightarrow B_{s_{i_{1}} \cdots s_{i_{k}}}, \quad\left(c_{1}, \ldots, c_{k}\right) \mapsto e^{c_{1} E_{i_{1}}} \cdots e^{c_{k} E_{i_{k}}{ }^{t}} B_{-} .
$$

5.2. Populations and Bruhat cells. Let $P$ be a population of critical points associated with weights $\boldsymbol{\Lambda}$, numbers $\boldsymbol{z}$. Let $T_{1}, \ldots, T_{r}$ be the polynomials defined by (44). Let $\boldsymbol{y}^{0}=\left(y_{1}^{0}, \ldots, y_{r}^{0}\right) \in P$ with $l_{i}=\operatorname{deg} y_{i}^{0}$ for $i \in\{1, \ldots, r\}$. Assume that the weight at infinity of $\boldsymbol{y}^{0}$,

$$
\Lambda_{\infty}=\sum_{i=1}^{n} \Lambda_{i}-\sum_{i=1}^{r} l_{i} \alpha_{i},
$$

is integral dominant, see Section 2. Such $\boldsymbol{y}^{0}$ exists according to MV1. For $w \in W$ consider the weight $w \cdot \Lambda_{\infty}$, where $w \cdot$ is the shifted action of $w$ on $\mathfrak{h}^{*}$. Write

$$
w \cdot \Lambda_{\infty}=\sum_{i=1}^{n} \Lambda_{i}-\sum_{i=1}^{r} l_{i}^{w} \alpha_{i}
$$

Set

$$
P_{w}=\left\{\boldsymbol{y}=\left(y_{1}, \ldots, y_{r}\right) \in P \mid \operatorname{deg} y_{i}=l_{i}^{w}, i=1, \ldots, r\right\} .
$$

Consider the trivial bundle $p^{\prime}:\left({ }^{t} G /{ }^{t} B_{-}\right) \times \mathbb{P}^{1} \rightarrow \mathbb{P}^{1}$ with connection $\nabla_{D_{y^{0}}}^{\prime}$. Consider the Bruhat cell decomposition of fibers of $p^{\prime}$.

Let $x_{0} \in \mathbb{C}$ be such that $T_{i}\left(x_{0}\right) \neq 0$ and $y_{i}^{0}\left(x_{0}\right) \neq 0$ for $i=1, \ldots, r$. The point $x_{0} \in \mathbb{C}$ is a regular point of the connection $\nabla_{D_{y^{0}}}^{\prime}$. Let

$$
\phi_{\boldsymbol{y}^{0}, x_{0}}: P \rightarrow{ }^{t} G /{ }^{t} B_{-}
$$

be the isomorphism defined in Section 4.3 
Theorem 5.1. For $w \in W$ we have

$$
\phi_{\boldsymbol{y}^{0}, x_{0}}\left(P_{w}\right)=B_{w^{-1}} .
$$

Corollary 5.3. Let $\Lambda_{1}, \ldots, \Lambda_{n}, \Lambda_{\infty}$ be integral dominant $\mathfrak{g}$-weights. Let $z_{1}, \ldots, z_{n}$ be distinct complex numbers. Let $w \in W$. Consider the master function $\Phi\left(\boldsymbol{t} ; \boldsymbol{z}, \boldsymbol{\Lambda}, w \cdot \Lambda_{\infty}\right)$. Let $K$ be a connected component of the critical set of the master function. For each $\boldsymbol{t} \in K$ consider the tuple $\boldsymbol{y}_{\boldsymbol{t}} \in(\mathbb{C}[x])^{r}$ of monic polynomials representing the critical point $\boldsymbol{t}$. Then the closure of the set $\left\{\boldsymbol{y}_{\boldsymbol{t}} \mid \boldsymbol{t} \in K\right\}$ is an $l(w)$-dimensional cell.

\subsection{Proof of Theorem 5.1.}

Lemma 5.4. For $w \in W$, the subset $B_{w} \times \mathbb{P}^{1} \subset\left({ }^{t} G /{ }^{t} B_{-}\right) \times \mathbb{P}^{1}$ is invariant with respect to the connection $\nabla_{D_{y^{0}}}^{\prime}$.

Proof. Let $Y$ be the ${ }^{t} G$-valued solution of the equation $D_{\boldsymbol{y}^{0}} Y=0$ such that $Y\left(x_{0}\right)=$ id. Then $Y(x) \in{ }^{t} B_{-}$for all $x$. The horizontal sections of $\nabla_{D_{y^{0}}}^{\prime}$ have the form $x \mapsto$ $\left(Y(x) u^{t} B_{-}\right) \times x$ for a suitable element $u \in{ }^{t} G$. Hence if $u{ }^{t} B_{-} \in B_{w}$, then $Y(x) u^{t} B_{-} \in$ $B_{w}$ for all $x$.

Let $w=s_{i_{k}} \cdots s_{i_{1}}$ be a reduced decomposition of $w \in W$. For $b=1, \ldots, k$ set

$$
\left(s_{i_{b}} \cdots s_{i_{1}}\right) \cdot \Lambda_{\infty}=\sum_{i=1}^{n} \Lambda_{i}-\sum_{i=1}^{r} l_{i}^{b} \alpha_{i} .
$$

From [BGG] it follows that $l_{i_{1}}^{1}>l_{i_{1}}$ and $l_{i_{b}}^{b}>l_{i_{b}}^{b-1}$ for $b=2, \ldots, k$.

For $b=1, \ldots, k$ define by induction on $b$ a family of tuples of polynomials depending on complex parameters $c_{1}, \ldots, c_{b}$. Namely, let $\tilde{y}_{i_{1}}$ be a polynomial satisfying equation

$$
W\left(y_{i_{1}}^{0}, \tilde{y}_{i_{1}}\right)=T_{i_{1}} \prod_{j, j \neq i_{1}}\left(y_{j}^{0}\right)^{-a_{i_{1}, j}}
$$

We fix $\tilde{y}_{i_{1}}$ assuming that the coefficient of $x^{l_{i_{1}}}$ in $\tilde{y}_{i_{1}}$ is equal to zero. Set $\boldsymbol{y}^{1 ; c_{1}}=$ $\left(y_{1}^{1 ; c_{1}}, \ldots, y_{r}^{1 ; c_{1}}\right)$, where

$$
y_{i_{1}}^{1 ; c_{1}}(x)=\tilde{y}_{i_{1}}(x)+c_{1} y_{i_{1}}^{0}(x) \quad \text { and } y_{j}^{1 ; c_{1}}(x)=y_{j}^{0}(x) \text { for } j \neq i_{1} .
$$

Assume that the family $\boldsymbol{y}^{b-1 ; c_{1}, \ldots, c_{b-1}}$ is already defined. Let $\tilde{y}_{i_{b}}{ }^{b-1 ; c_{1}, \ldots, c_{b-1}}$ be a polynomial satisfying equation

$$
W\left(y_{i_{b}}^{b-1 ; c_{1}, \ldots, c_{b-1}}, \tilde{y}_{i_{b}}^{b-1 ; c_{1}, \ldots, c_{b-1}}\right)=T_{i_{b}} \prod_{j, j \neq i_{b}}\left(y_{j}^{b-1 ; c_{1}, \ldots, c_{b-1}}\right)^{-a_{i_{b}, j}} .
$$

We fix $\tilde{y}_{i_{d}}^{d-1 ; c_{1}, \ldots, c_{d-1}}$ assuming that the coefficient of $x^{l_{i}^{d-1}}$ in $\tilde{y}_{i_{d}}^{d-1 ; c_{1}, \ldots, c_{d-1}}$ is equal to zero. Set $\boldsymbol{y}^{b ; c_{1}, \ldots, c_{b}}=\left(y_{1}^{b ; c_{1}, \ldots, c_{b}}, \ldots, y_{r}^{b ; c_{1}, \ldots, c_{b}}\right)$, where

$$
y_{i_{b}}^{b ; c_{1}, \ldots, c_{b}}(x)=\tilde{y}_{i_{b}}^{b-1 ; c_{1}, \ldots, c_{b}}(x)+c_{b} y_{i_{b}}^{b-1 ; c_{1}, \ldots, c_{b-1}}(x)
$$


and

$$
y_{j}^{b ; c_{1}, \ldots, c_{b}}(x)=y_{j}^{b-1 ; c_{1}, \ldots, c_{b-1}}(x) \text { for } j \neq i_{b} .
$$

The $b$-th family is obtained from the $(b-1)$-st family by the generation procedure in the $i_{b}$-th direction, see Section 2.5. For any $c_{1}, \ldots, c_{k}$ the tuple $\boldsymbol{y}^{k ; c_{1}, \ldots, c_{k}}$ lies in $P$.

For any $c_{1}, \ldots, c_{k}$ and any $i \in\{1, \ldots, r\}$, we have

$$
\operatorname{deg} y_{i}^{k ; c_{1}, \ldots, c_{k}}(x)=l_{i}^{w} \text {. }
$$

Set

$$
P^{\left[i_{1}, \ldots, i_{k}\right]}=\left\{\boldsymbol{y}^{k ; c_{1}, \ldots, c_{k}} \mid c_{1}, \ldots, c_{k} \in \mathbb{C}\right\} .
$$

Proposition 5.5. We have

$$
\phi_{\boldsymbol{y}^{0}, x_{0}}\left(P^{\left[i_{1}, \ldots, i_{k}\right]}\right)=B_{w^{-1}}
$$

Proof of Proposition 5.5. Let $D_{\boldsymbol{y}^{k ; c_{1}, \ldots, c_{k}}}$ be the Miura oper associated with the tuple $\boldsymbol{y}^{k ; c_{1}, \ldots, c_{k}}$, then

$$
\begin{gathered}
D_{\boldsymbol{y}^{k ; c_{1}, \ldots, c_{k}}}=\exp \left(\operatorname{adlog}^{\prime}\left(\frac{y_{i_{k}}^{k ; c_{1}, \ldots, c_{k}}}{y_{i_{k}}^{k-1 ; c_{1}, \ldots, c_{k-1}}}\right) E_{i_{k}}\right) \ldots \\
\quad \exp \left(\operatorname{ad} \log ^{\prime}\left(\frac{y_{i_{2}}^{2 ; c_{1}, c_{2}}}{y_{i_{2}}^{1 ; c_{1}}}\right) E_{i_{2}}\right) \exp \left(\operatorname{adlog}^{\prime}\left(\frac{y_{i_{1}}^{1 ; c_{1}}}{y_{i_{1}}^{0}}\right) E_{i_{1}}\right) \cdot D_{\boldsymbol{y}^{0}}= \\
\exp \left(-\operatorname{ad}\left(\frac{T_{i_{k}} \prod_{j, j \neq i_{k}}\left(y_{j}^{k-1 ; c_{1}, \ldots, c_{k-1}}\right)^{-a_{i_{k}, j}}}{y_{i_{k}}^{k ; c_{1}, \ldots, c_{k}} y_{i_{k}}^{k-1 ; c_{1}, \ldots, c_{k-1}}}\right) E_{i_{k}}\right) \ldots \\
\exp \left(-\operatorname{ad}\left(\frac{T_{i_{2}} \prod_{j, j \neq i_{2}}\left(y_{j}^{1 ; c_{1}}\right)^{-a_{i_{2}, j}}}{y_{i_{2}}^{2 ; c_{1}, c_{2}} y_{i_{2}}^{1 ; c_{1}}}\right) E_{i_{2}}\right) \exp \left(-\operatorname{ad}\left(\frac{T_{i_{1}} \prod_{j, j \neq i_{1}}\left(y_{j}^{0}\right)^{-a_{i_{1}, j}}}{y_{i_{1}}^{1 ; c_{1}} y_{i_{1}}^{0}}\right) E_{i_{1}}\right) \cdot D_{\boldsymbol{y}^{0}},
\end{gathered}
$$

see Theorem 3.1 .

Introduce the rational map

$$
g: \mathbb{C}^{k+1} \rightarrow \mathbb{C}^{k}, \quad\left(x ; c_{1}, \ldots, c_{k}\right) \mapsto\left(g_{1}\left(x ; c_{1}\right), \ldots, g_{k}\left(x ; c_{1}, \ldots, c_{k}\right)\right)
$$

where

$$
\begin{gathered}
g_{1}\left(x ; c_{1}\right)=\frac{T_{i_{1}}(x) \prod_{j, j \neq i_{1}}\left(y_{j}^{0}(x)\right)^{-a_{i_{1}, j}}}{y_{i_{1}}^{1 ; c_{1}}(x) y_{i_{1}}^{0}(x)}, \\
g_{b}\left(x ; c_{1}, \ldots, c_{b}\right)=\frac{T_{i_{b}}(x) \prod_{j, j \neq i_{b}}\left(y_{j}^{b-1 ; c_{1}, \ldots, c_{b-1}}(x)\right)^{-a_{i_{b}, j}}}{y_{i_{b}}^{b ; c_{1}, \ldots, c_{b}}(x) y_{i_{b}}^{b-1 ; c_{1}, \ldots, c_{b-1}}(x)}
\end{gathered}
$$


for $b=2, \ldots, k$. From (14) it follows that the tuple $\boldsymbol{y}^{k ; c_{1}, \ldots, c_{k}}$ corresponds to the rational section

$$
S_{\left(c_{1}, \ldots, c_{k}\right)}: x \mapsto f_{i_{1}, \ldots, i_{k}}\left(g\left(x, c_{1}, \ldots, c_{k}\right)\right) \times x
$$

of the bundle $p^{\prime}$. This section is horizontal with respect to the connection $\nabla_{D_{y^{0}}}^{\prime}$, and we have

$$
\phi_{\boldsymbol{y}^{0}, x}\left(\boldsymbol{y}^{k ; c_{1}, \ldots, c_{k}}\right)=f_{i_{1}, \ldots, i_{k}}\left(g\left(x, c_{1}, \ldots, c_{k}\right)\right)
$$

This means that

$$
\phi_{\boldsymbol{y}^{0}, x_{0}}\left(P^{\left[i_{1}, \ldots, i_{k}\right]}\right) \subset B_{w^{-1}}
$$

It remains to show that every point in $B_{w^{-1}}$ is the limit of points of $\phi_{\boldsymbol{y}^{0}, x_{0}}\left(P^{\left[i_{1}, \ldots, i_{k}\right]}\right)$, where the limit is taken in the sense of the limit in Corollary [5.2, but that statement follows from

Lemma 5.6. Let $x_{0} \in \mathbb{C}$ be such that $T_{i}\left(x_{0}\right) \neq 0$ and $y_{i}^{0}\left(x_{0}\right) \neq 0$ for $i=1, \ldots, r$. Then for any $\left(c_{1}^{1}, \ldots, c_{k}^{1}\right) \in(\mathbb{C}-0)^{k}$ there exists a unique $\left(c_{1}^{2}, \ldots, c_{k}^{2}\right) \in \mathbb{C}^{k}$ such that

$$
\left(c_{1}^{1}, \ldots, c_{k}^{1}\right)=g\left(x_{0} ; c_{1}^{2}, \ldots, c_{k}^{2}\right) .
$$

The proposition is proved.

Theorem 5.1 is a direct corollary of Proposition 5.5 .

\section{Solutions of Differential Equations}

As we observed earlier, the Miura opers, associated with a population of critical points, help to study the structure of the population. In addition to that it turns out that for a Miura oper $D$ associated with a critical point of a population, all solutions of the differential equation $D Y=0$ with values in the corresponding group can be written explicitly in terms of critical points composing the population.

First we give formulas for solutions of the equation $D Y=0$ for opers associated with Lie algebras of types $A_{r}, B_{r}$, and then consider more general formulas for solutions which do not use the structure of the Lie algebra.

In this section $\mathfrak{g}=\mathfrak{g}(A)$ is a simple Lie algebra with Cartan matrix $A=\left(a_{i, j}\right)$.

6.1. Elimination of polynomials $T_{i}$. Let $B=\left(b_{i, j}\right)$ be the matrix inverse to $A$.

Let $D$ be the Miura ${ }^{t} G$-oper associated with weights $\boldsymbol{\Lambda}$, numbers $\boldsymbol{z}$, and a tuple $\boldsymbol{y}=\left(y_{1}, \ldots, y_{r}\right)$. Introduce polynomials $T_{1}(x), \ldots, T_{r}(x)$ by formulas (4). Introduce a tuple of functions $\overline{\boldsymbol{y}}=\left(\bar{y}_{1}, \ldots, \bar{y}_{r}\right)$ by

$$
\bar{y}_{i}=y_{i} \prod_{l=1}^{r} T_{l}^{-b_{i, l}} .
$$


Lemma 6.1. We have $D=\partial+I+V$ where

$$
V=\sum_{j=1}^{r} \log ^{\prime}\left(\bar{y}_{j}\right) H_{j}
$$

Proof. If $V$ is given by (16), then

$$
\left\langle{ }^{t} \alpha_{i}, V\right\rangle=\sum_{j=1}^{r} \log ^{\prime}\left(\bar{y}_{j}\right)\left\langle{ }^{t} \alpha_{i},{ }^{t} \alpha_{j}^{\vee}\right\rangle=\sum_{j=1}^{r} \log ^{\prime}\left(\bar{y}_{j}{ }^{a_{i, j}}\right)=\log ^{\prime}\left(T_{i}^{-1} \prod_{j=1}^{r} y_{j}{ }^{a_{i, j}}\right) .
$$

Assume that $\boldsymbol{y}$ represents a critical point of the master function (11) associated with parameters $\boldsymbol{z}, \boldsymbol{\Lambda}, \Lambda_{\infty}$.

Let $\boldsymbol{i}=\left[i_{1}, \ldots, i_{k}\right], i_{j} \in\{1, \ldots, r\}$, be a sequence of natural numbers. Let $\boldsymbol{y}^{\left[i_{1}\right]}=$ $\left(y_{1}^{\left[i_{1}\right]}, \ldots, y_{r}^{\left[i_{1}\right]}\right), \boldsymbol{y}^{\left[i_{1}, i_{2}\right]}=\left(y_{1}^{\left[i_{1}, i_{2}\right]}, \ldots, y_{r}^{\left[i_{1}, i_{2}\right]}\right), \ldots, \boldsymbol{y}^{\left[i_{1}, \ldots, i_{k}\right]}=\left(y_{1}^{\left[i_{1}, \ldots, i_{k}\right]}, \ldots, y_{r}^{\left[i_{1}, \ldots, i_{k}\right]}\right)$ be a sequence of tuples associated with the critical point $\boldsymbol{y}$ and the sequence of indices $\boldsymbol{i}$, see Section [2.5. Introduce functions $\bar{y}_{i}^{\left[i_{1}, \ldots, i_{l}\right]}$ by

$$
\bar{y}_{i}^{\left[i_{1}, \ldots, i_{l}\right]}=y_{i}^{\left[i_{1}, \ldots, i_{l}\right]} \prod_{l=1}^{r} T_{l}^{-b_{i, l}} .
$$

Lemma 6.2. We have

$$
W\left(\bar{y}_{i_{1}}, \bar{y}_{i_{1}}^{\left[i_{1}\right]}\right)=\prod_{j, j \neq i_{1}} \bar{y}_{j}^{-a_{i_{1}, j}}
$$

and $\bar{y}_{j}^{\left[i_{1}\right]}=\bar{y}_{j}$ for $j \neq i_{1} ; \quad$ for $l=2, \ldots, k$, we have

$$
W\left(\bar{y}_{i_{l}}^{\left[i_{1}, \ldots, i_{l-1}\right]}, \bar{y}_{i_{l}}^{\left[i_{1}, \ldots, i_{l}\right]}\right)=\prod_{j, j \neq i_{l}}\left(\bar{y}_{j}^{\left[i_{1}, \ldots, i_{l-1}\right]}\right)^{-a_{i_{l}, j}}
$$

and $\bar{y}_{j}^{\left[i_{1}, \ldots, i_{l}\right]}=\bar{y}_{j}^{\left[i_{1}, \ldots, i_{l-1}\right]}$ for $j \neq i_{l}$.

The sequence of tuples $\overline{\boldsymbol{y}}^{\left[i_{1}\right]}=\left(\bar{y}_{1}^{\left[i_{1}\right]}, \ldots, \bar{y}_{r}^{\left[i_{1}\right]}\right), \overline{\boldsymbol{y}}^{\left[i_{1}, i_{2}\right]}=\left(\bar{y}_{1}^{\left[i_{1}, i_{2}\right]}, \ldots, \bar{y}_{r}^{\left[i_{1}, i_{2}\right]}\right), \ldots$, $\overline{\boldsymbol{y}}^{\left[i_{1}, i_{2}, \ldots, i_{k}\right]}=\left(\bar{y}_{1}^{\left[i_{1}, i_{2}, \ldots, i_{k}\right]}, \ldots, \bar{y}_{r}^{\left[i_{1}, i_{2}, \ldots, i_{k}\right]}\right)$ will be called the sequence of reduced tuples associated with the critical point $\boldsymbol{y}$ and the sequence of indices $\boldsymbol{i}$.

The sequence of functions $\bar{y}_{i_{1}}^{\left[i_{1}\right]}, \bar{y}_{i_{2}}^{\left[i_{1}, i_{2}\right]}, \ldots, \bar{y}_{i_{k}}^{\left[i_{1}, i_{2}, \ldots, i_{k}\right]}$, each defined up to multiplication by a non-zero number, will be called the reduced diagonal sequence of functions associated with the critical point $\boldsymbol{y}$ and the sequence of indices $\boldsymbol{i}$.

The reduced diagonal sequence of functions determine the sequence of tuples $\overline{\boldsymbol{y}}^{\left[i_{1}\right]}$, $\overline{\boldsymbol{y}}^{\left[i_{1}, i_{2}\right]}, \ldots, \overline{\boldsymbol{y}}^{\left[i_{1}, i_{2}, \ldots, i_{k}\right]}$ uniquely.

In the next sections we use the following lemma.

\section{Lemma 6.3.}


- Consider the product $\prod_{j=1}^{r} \bar{y}_{j}{ }^{-H_{j}}$ as a function of $x$ with values in the group ${ }^{t} G$. Then

$$
\prod_{j=1}^{r} \bar{y}_{j}{ }^{-H_{j}}=\prod_{j=1}^{r} y_{j}{ }^{-H_{j}} T_{j}^{w_{j}}
$$

where $w_{1}, \ldots, w_{r} \in{ }^{t} \mathfrak{h}$ are the fundamental coweights, i.e. $\left\langle{ }^{t} \alpha_{i}, w_{j}\right\rangle=\delta_{i, j}$.

- Let $D=\partial+I+V$ be the Miura oper with $V$ given by formula (16). Define

$$
\bar{D}=\partial+\sum_{j=1}^{r}\left(\prod_{l=1}^{r} \bar{y}_{l}^{-a_{j, l}}\right) F_{j} .
$$

Then

$$
D\left(\prod_{j=1}^{r} \bar{y}_{j}^{-H_{j}}\right)=\left(\prod_{j=1}^{r} \bar{y}_{j}^{-H_{j}}\right) \bar{D}
$$

6.2. The $A_{r}$ critical points and $A_{r}$ opers. The Lie algebra $s l_{r+1}$ is of $A_{r}$-type. The Langlands dual to $s l_{r+1}$ is $s l_{r+1}$. Let $F_{1}, \ldots, F_{r}, H_{1}, \ldots, H_{r}, E_{1}, \ldots, E_{r}$ be the Chevalley generators of $s l_{r+1}$. Let $w_{1}, \ldots, w_{r}$ be the fundamental coweights of $s l_{r+1}$.

We start with two examples.

Let $\mathfrak{g}=s l_{2}$. Let $\boldsymbol{y}=\left(y_{1}\right)$ represent a critical point of the $s l_{2}$ master function (11) associated with parameters $\boldsymbol{z}, \boldsymbol{\Lambda}, \Lambda_{\infty}$. Introduce the function $\bar{y}_{1}=y_{1} T_{1}^{-1 / 2}$, see (15). Then the Miura oper associated with $\boldsymbol{y}$ has the form

$$
D=\partial+F_{1}+\log ^{\prime}\left(\bar{y}_{1}\right) H_{1} .
$$

Let $\bar{y}_{1}^{[1]}$ be the reduced diagonal sequence of functions associated with $\boldsymbol{y}$ and the sequence of indices [1], in other words, $W\left(\bar{y}_{1}, \bar{y}_{1}^{[1]}\right)=1$. Then

$$
Y=\bar{y}_{1}^{-H_{1}} e^{\bar{y}_{1}^{[1]} F_{1}}
$$

is a solution of the differential equation $D Y=0$ with values in $\operatorname{SL}(2, \mathbb{C})$. Indeed,

$$
D Y=\bar{y}_{1}^{-H_{1}}\left(\partial+\frac{1}{\left(\bar{y}_{1}\right)^{2}} F_{1}\right) e^{\frac{\bar{y}_{1}^{[1]}}{\bar{y}_{1}} F_{1}}=Y\left(\partial+\left(\left(\frac{\bar{y}_{1}^{[1]}}{\bar{y}_{1}}\right)^{\prime}+\frac{1}{\left(\bar{y}_{1}\right)^{2}}\right) F_{1}\right) \mathrm{id}=Y \partial \mathrm{id}=0 .
$$

Let $\mathfrak{g}=s l_{3}$. Let $\boldsymbol{y}=\left(y_{1}, y_{2}\right)$ represent a critical point of the $s l_{3}$ master function (11) associated with parameters $\boldsymbol{z}, \boldsymbol{\Lambda}, \Lambda_{\infty}$. Introduce the functions $\bar{y}_{1}=y_{1} T_{1}{ }^{-2 / 3} T_{2}{ }^{-1 / 3}$, $\bar{y}_{2}=y_{2} T_{1}^{-1 / 3} T_{2}{ }^{-2 / 3}$, see (15). Then the Miura oper associated with $\boldsymbol{y}$ has the form

$$
D=\partial+F_{1}+F_{2}+\log ^{\prime}\left(\bar{y}_{1}\right) H_{1}+\log ^{\prime}\left(\bar{y}_{2}\right) H_{2} .
$$


Let $\bar{y}_{1}^{[1]}, \bar{y}_{2}^{[1,2]}$ be the reduced diagonal sequence of functions associated with $\boldsymbol{y}$ and the sequence of indices $[1,2]$, in other words,

$$
W\left(\bar{y}_{1}, \bar{y}_{1}^{[1]}\right)=\bar{y}_{2}, \quad W\left(\bar{y}_{2}, \bar{y}_{2}^{[1,2]}\right)=\bar{y}_{1}^{[1]} .
$$

Let $\bar{y}_{2}^{[2]}$ be the reduced diagonal sequence of functions associated with $\boldsymbol{y}$ and the sequence of indices [2], in other words, $W\left(\bar{y}_{2}, \bar{y}_{2}^{[2]}\right)=\bar{y}_{1}$. Then

$$
Y=\bar{y}_{1}^{-H_{1}} \bar{y}_{2}^{-H_{2}} e^{\frac{\bar{y}_{1}^{[1]}}{\bar{y}_{1}} F_{1}} e^{\frac{\bar{y}_{2}^{[1,2]}}{\bar{y}_{2}}\left[F_{2}, F_{1}\right]} e^{\frac{\bar{y}_{2}^{[2]}}{\bar{y}_{2}} F_{2}}
$$

is a solution of the differential equation $D Y=0$ with values in $\operatorname{SL}(3, \mathbb{C})$. Indeed, by Lemma 6.3 it suffices to show that

$$
\bar{Y}=e^{\frac{\bar{y}_{1}^{[1]}}{\bar{y}_{1}} F_{1}} e^{\frac{\bar{y}_{2}^{[1,2]}}{\bar{y}_{2}}\left[F_{2}, F_{1}\right]} e^{\frac{\bar{y}_{2}^{[2]}}{\bar{y}_{2}} F_{2}}
$$

is a solution of the differential equation $\bar{D} Y=0$ where

$$
\bar{D}=\partial+\frac{\bar{y}_{2}}{\left(\bar{y}_{1}\right)^{2}} F_{1}+\frac{\bar{y}_{1}}{\left(\bar{y}_{2}\right)^{2}} F_{2} .
$$

Indeed,

$$
\begin{gathered}
\bar{D} \bar{Y}=e^{\frac{\bar{y}_{1}^{[1]}}{\bar{y}_{1}} F_{1}}\left(\partial+\left(\left(\frac{\bar{y}_{1}^{[1]}}{\bar{y}_{1}}\right)^{\prime}+\frac{1}{\left(\bar{y}_{1}\right)^{2}}\right) F_{1}+\frac{\bar{y}_{1}^{[1]}}{\left(\bar{y}_{2}\right)^{2}}\left[F_{2}, F_{1}\right]+\frac{\bar{y}_{1}}{\left(\bar{y}_{2}\right)^{2}} F_{2}\right) e^{\frac{\bar{y}_{2}^{[1,2]}}{\bar{y}_{2}}\left[F_{2}, F_{1}\right]} e^{\frac{\bar{y}_{2}^{[2]}}{\bar{y}_{2}} F_{2}} \\
=e^{\frac{\bar{y}_{1}^{[1]}}{\bar{y}_{1}} F_{1}}\left(\partial+\frac{\bar{y}_{1}^{[1]}}{\left(\bar{y}_{2}\right)^{2}}\left[F_{2}, F_{1}\right]+\frac{\bar{y}_{1}}{\left(\bar{y}_{2}\right)^{2}} F_{2}\right) e^{\frac{\bar{y}_{2}^{[1,2]}}{\bar{y}_{2}}\left[F_{2}, F_{1}\right]} e^{\frac{\bar{y}_{2}^{[2]}}{\bar{y}_{2}} F_{2}} \\
\left.=e^{\frac{\bar{y}_{1}^{[1]}}{\bar{y}_{1}} F_{1}} e^{\frac{\bar{y}_{2}^{[1,2]}}{\bar{y}_{2}}\left[F_{2}, F_{1}\right]}\left(\partial+\left(\frac{\bar{y}_{2}^{[1,2]}}{\bar{y}_{2}}\right)^{\prime}+\frac{\bar{y}_{1}^{[1]}}{\left(\bar{y}_{2}\right)^{2}}\right)\left[F_{2}, F_{1}\right]+\frac{\bar{y}_{1}}{\left(\bar{y}_{2}\right)^{2}} F_{2}\right) e^{\frac{\bar{y}_{2}^{[2]}}{\bar{y}_{2}} F_{2}} \\
=e^{\frac{\bar{y}_{1}^{[1]}}{\bar{y}_{1}} F_{1}} e^{\frac{\bar{y}_{2}^{[1,2]}}{\bar{y}_{2}}\left[F_{2}, F_{1}\right]}\left(\partial+\frac{\bar{y}_{1}}{\left(\bar{y}_{2}\right)^{2}} F_{2}\right) e^{\frac{\bar{y}_{2}^{[2]}}{\bar{y}_{2}} F_{2}} \\
=e^{\frac{\bar{y}_{1}^{[1]}}{\bar{y}_{1}} F_{1}} e^{\frac{\bar{y}_{2}^{[1,2]}}{\bar{y}_{2}}\left[F_{2}, F_{1}\right]} e^{\frac{\bar{y}_{2}^{2[2]}}{\bar{y}_{2}} F_{2}}\left(\partial+\left(\left(\frac{\bar{y}_{2}^{[2]}}{\bar{y}_{2}}\right)^{\prime}+\frac{\bar{y}_{1}}{\left(\bar{y}_{2}\right)^{2}}\right) F_{2}\right) \mathrm{id} \\
=e^{\frac{\bar{y}_{1}^{[1]}}{\bar{y}_{1}} F_{1}} e^{\frac{\bar{y}_{2}^{[1,2]}}{\bar{y}_{2}}\left[F_{2}, F_{1}\right]} e^{\frac{\bar{y}_{2}^{[2]}}{\bar{y}_{2}} F_{2}} \partial \mathrm{id}=0 .
\end{gathered}
$$

Now consider the general case. Let $\mathfrak{g}=s l_{r+1}$. Let $\boldsymbol{y}=\left(y_{1}, \ldots, y_{r}\right)$ represent a critical point of the $s l_{r+1}$ master function (11) associated with parameters $\boldsymbol{z}, \boldsymbol{\Lambda}, \Lambda_{\infty}$. Introduce the functions $\bar{y}_{1}, \ldots, \bar{y}_{r}$ by formula (15), where $B=\left(b_{i, j}\right)$ is the matrix inverse to the Cartan matrix of $s l_{r+1}$. Then the Miura oper associated with $\boldsymbol{y}$ has the form

$$
D=\partial+\sum_{j=1}^{r} F_{j}+\sum_{j=1}^{r} \log ^{\prime}\left(\bar{y}_{j}\right) H_{j} .
$$


For $i=1, \ldots, r$, let $y_{i}^{[i]}, y_{i+1}^{[i, i+1]}, \ldots, y_{r}^{[i, \ldots, r]}$ be the diagonal sequence of polynomials associated with $\boldsymbol{y}$ and the sequence of indices $[i, i+1, \ldots, r]$, in other words,

$$
\begin{gathered}
W\left(y_{i}, y_{i}^{[i]}\right)=T_{i} y_{i-1} y_{i+1}, \quad W\left(y_{i+1}, y_{i+1}^{[i, i+1]}\right)=T_{i+1} y_{i}^{[i]} \bar{y}_{i+2}, \ldots, \\
W\left(y_{r-1}, y_{r-1}^{[i, \ldots, r-1]}\right)=T_{r-1} y_{r-2}^{[i, \ldots, r-2]} y_{r}, \quad W\left(y_{r}, y_{r}^{[i, \ldots, r]}\right)=T_{r} y_{r-1}^{[i, \ldots, r-1]} .
\end{gathered}
$$

Define $r+1$ functions $Y_{0}, Y_{1}, \ldots, Y_{r}$ of $x$ with values in $\mathrm{SL}(r+1, \mathbb{C})$ by the formulas

$$
Y_{0}=\prod_{j=1}^{r} y_{j}{ }^{-H_{j}} T_{j}{ }^{w_{j}}, \quad Y_{i}=\prod_{j=i}^{r} e^{\frac{y_{j}^{[i, \ldots, j]}}{y_{j}}\left[F_{j},\left[F_{j-1},\left[\ldots,\left[F_{i+1}, F_{i}\right] \ldots\right]\right]\right]}, \quad \text { for } i>0 .
$$

Note that inside each product the factors commute.

Theorem 6.1. The product $Y_{0} Y_{1} \ldots Y_{r}$ is a solution of the differential equation $D Y=0$ with values in $\mathrm{SL}(r+1, \mathbb{C})$ where $D$ is given by (20).

Note that if $Y(x)$ is a solution of the equation $D Y=0$ and $g \in \mathrm{SL}(r+1, \mathbb{C})$, then $Y(x) g$ is a solution too.

The proof of the theorem is straightforward. One uses Lemma 6.3 and then shows that

$$
\left(\partial+\sum_{j=i}^{r} \frac{\bar{y}_{j-1} \bar{y}_{j+1}}{\bar{y}_{j}{ }^{2}} F_{j}\right) Y_{i}=Y_{i}\left(\partial+\sum_{j=i+1}^{r} \frac{\bar{y}_{j-1} \bar{y}_{j+1}}{\bar{y}_{j}{ }^{2}} F_{j}\right)
$$

for $i=1, \ldots, r$. In this formula we set $\bar{y}_{0}=\bar{y}_{r+1}=1$.

6.3. The $B_{r}$ critical points and $C_{r}$ opers. Consider the root system of type $B_{r}$. Let $\alpha_{1}, \ldots, \alpha_{r-1}$ be the long simple roots and $\alpha_{r}$ the short one. We have

$$
\left(\alpha_{r}, \alpha_{r}\right)=2, \quad\left(\alpha_{i}, \alpha_{i}\right)=4, \quad\left(\alpha_{i}, \alpha_{i+1}\right)=-2, \quad i=1, \ldots, r-1,
$$

and all other scalar products are equal to zero. The root system $B_{r}$ corresponds to the Lie algebra $s_{2 r+1}$. Let $\mathfrak{h}_{B}$ be its Cartan subalgebra.

Consider the root system of type $C_{r}$. The root system $C_{r}$ corresponds to the Lie algebra $s p_{2 r}$. Let $F_{1}, \ldots, F_{r}, H_{1}, \ldots, H_{r}, E_{1}, \ldots, E_{r}$ be its Chevalley generators and $w_{1}, \ldots, w_{r}$ the fundamental coweights. The symplectic group $\operatorname{Sp}(2 r, \mathbb{C})$ is the simply connected group with Lie algebra $s p_{2 r}$.

The Lie algebras $s o_{2 r+1}$ and $s p_{2 r}$ are Langlands dual.

We consider also the root system of type $A_{2 r-1}$ with simple roots $\alpha_{1}^{A}, \ldots, \alpha_{2 r-1}^{A}$. The root system $A_{2 r-1}$ corresponds to the Lie algebra $s l_{2 r}$. We denote $\mathfrak{h}_{A}$ its Cartan subalgebra.

We have a map $\mathfrak{h}_{B}^{*} \rightarrow \mathfrak{h}_{A}^{*}, \Lambda \mapsto \Lambda^{A}$, where $\Lambda^{A}$ is defined by

$$
\left\langle\Lambda^{A},\left(\alpha_{i}^{A}\right)^{\vee}\right\rangle=\left\langle\Lambda^{A},\left(\alpha_{2 r-i}^{A}\right)^{\vee}\right\rangle=\left\langle\Lambda,\left(\alpha_{i}\right)^{\vee}\right\rangle, \quad i=1, \ldots, r .
$$


Let $\Lambda_{1}, \ldots, \Lambda_{n} \in \mathfrak{h}_{B}^{*}$ be dominant integral $s o_{2 r+1}$-weights, $z_{1}, \ldots, z_{n}$ complex numbers. Let the polynomials $T_{1}, \ldots, T_{r}$ be given by (4). Remind that an $r$-tuple of polynomials $\boldsymbol{y}$ represents a critical point of a master function associated with $s o_{2 r+1}, \Lambda_{1}, \ldots, \Lambda_{n}$, $z_{1}, \ldots, z_{n}$, if and only if $\boldsymbol{y}$ is generic with respect to weights $\Lambda_{1}, \ldots, \Lambda_{n}$ of $s o_{2 r+1}$, and points $z_{1}, \ldots z_{n}$ and there exist polynomials $\tilde{y}_{i}, i=1, \ldots, r$, such that

$$
\begin{aligned}
& W\left(y_{i}, \tilde{y}_{i}\right)=T_{i} y_{i-1} y_{i+1}, \quad i=1, \ldots, r-1, \\
& W\left(y_{r}, \tilde{y}_{r}\right)=T_{r} y_{r-1}^{2} .
\end{aligned}
$$

For an $r$-tuple of polynomials $\boldsymbol{y}=\left(y_{1}, \ldots, y_{r}\right)$, let $\boldsymbol{u}$ be the $2 r-1$-tuple of polynomials $\left(u_{1}, \ldots, u_{2 r-1}\right)=\left(y_{1}, \ldots, y_{r-1}, y_{r}, y_{r-1}, \ldots, y_{1}\right)$.

Lemma 6.4 ([MV1]). An r-tuple $\boldsymbol{y}$ represents a critical point of the $s_{2 r+1}$ master function associated with $\Lambda_{1}, \ldots, \Lambda_{n}, z_{1}, \ldots, z_{n}$, if and only if the $2 r-1$-tuple of polynomials $\boldsymbol{u}$ represents a critical point of the sl $l_{2 r}$ master function associated with $\Lambda_{1}^{A}, \ldots, \Lambda_{n}^{A}$, $z_{1}, \ldots, z_{n}$.

We start with an example. Let $\boldsymbol{y}=\left(y_{1}, y_{2}\right)$ represent a critical point of the $s_{3}$ master function (11) associated with parameters $\boldsymbol{z}, \boldsymbol{\Lambda}, \Lambda_{\infty}$. Set $\bar{y}_{1}=y_{1} T_{1}{ }^{-1} T_{2}{ }^{-1 / 2}$, $\bar{y}_{2}=y_{2} T_{1}^{-1} T_{2}^{-1}$, see (15).

Let $\boldsymbol{u}=\left(u_{1}, u_{2}, u_{3}\right)=\left(y_{1}, y_{2}, y_{1}\right)$ be the tuple representing the corresponding $s l_{4}$ critical point. Set $\overline{\boldsymbol{u}}=\left(\bar{u}_{1}, \bar{u}_{2}, \bar{u}_{3}\right)=\left(\bar{y}_{1}, \bar{y}_{2}, \bar{y}_{1}\right)$.

Let $\bar{y}_{1}^{[1]}, \bar{y}_{2}^{[1,2]}$ be the $s_{3}$ reduced diagonal sequence of functions associated with $\boldsymbol{y}$ and the sequence of indices $[1,2]$, in other words,

$$
W\left(\bar{y}_{1}, \bar{y}_{1}^{[1]}\right)=\bar{y}_{2}, \quad W\left(\bar{y}_{2}, \bar{y}_{2}^{[1,2]}\right)=\left(\bar{y}_{1}^{[1]}\right)^{2} .
$$

Let $\bar{y}_{2}^{[2]}$ be the ${s o_{3}}_{3}$ reduced diagonal sequence of functions associated with $\boldsymbol{y}$ and the sequence of indices [2], in other words,

$$
W\left(\bar{y}_{2}, \bar{y}_{2}^{[2]}\right)=\left(\bar{y}_{1}\right)^{2}
$$

Let $\bar{u}_{1}^{[1]}=\bar{y}_{1}^{[1]}, \bar{u}_{2}^{[1,2]}$ be the $s l_{4}$ reduced diagonal sequence of functions associated with $\boldsymbol{u}$ and the sequence of indices [1,2], in other words,

$$
W\left(\bar{y}_{1}, \bar{y}_{1}^{[1]}\right)=\bar{y}_{2}, \quad W\left(\bar{y}_{2}, \bar{u}_{2}^{[1,2]}\right)=\bar{y}_{1}^{[1]} \bar{y}_{1} \text {. }
$$

Then

$$
Y=\bar{y}_{1}^{-H_{1}} \bar{y}_{2}^{-H_{2}} e^{\frac{\bar{y}_{1}^{[1]}}{\bar{y}_{1}} F_{1}} e^{\frac{1}{2} \frac{\bar{y}_{2}^{[1,2]}}{\bar{y}_{2}}\left[\left[F_{2}, F_{1}\right], F_{1}\right]} e^{\frac{\bar{u}_{2}^{[1,2]}}{\bar{y}_{2}}\left[F_{2}, F_{1}\right]} e^{\frac{\bar{y}_{2}^{[2]}}{\bar{y}_{2}} F_{2}}
$$

is an $\operatorname{Sp}(4, \mathbb{C})$-valued solution of the differential equation $D Y=0$ where

$$
D=\partial+F_{1}+F_{2}+\log ^{\prime}\left(\bar{y}_{1}\right) H_{1}+\log ^{\prime}\left(\bar{y}_{2}\right) H_{2} .
$$


Indeed, denote the factors of $Y$ by $P_{1}, \ldots, P_{6}$ counting from the left. By Lemma 6.3 it suffices to show that the product $P_{3} P_{4} P_{5} P_{6}$ is a solution of the equation $\bar{D} Y=0$ where

$$
\bar{D}=\partial+\frac{\bar{y}_{2}}{\bar{y}_{2}^{2}} F_{1}+\frac{\bar{y}_{1}^{2}}{\bar{y}_{2}^{2}} F_{2} \text {. }
$$

We have

$$
\begin{aligned}
\bar{D} P_{3} P_{4} P_{5} P_{6} & =P_{3}\left(\partial+\frac{\bar{y}_{1} \bar{y}_{1}^{[1]}}{\bar{y}_{2}^{2}}\left[F_{2}, F_{1}\right]+\frac{1}{2} \frac{\left(\bar{y}_{1}^{[1]}\right)^{2}}{\bar{y}_{2}^{2}}\left[\left[F_{2}, F_{1}\right], F_{1}\right]+\frac{\bar{y}_{1}^{2}}{\bar{y}_{2}{ }^{2}} F_{2}\right) P_{4} P_{5} P_{6} \\
& =P_{3} P_{4}\left(\partial+\frac{\bar{y}_{1} \bar{y}_{1}^{[1]}}{\bar{y}_{2}^{2}}\left[F_{2}, F_{1}\right]+\frac{\bar{y}_{1}^{2}}{\bar{y}_{2}^{2}} F_{2}\right) P_{5} P_{6} \\
& =P_{3} P_{4} P_{5}\left(\partial+\frac{\bar{y}_{1}^{2}}{\bar{y}_{2}^{2}} F_{2}\right) P_{6}=P_{3} P_{4} P_{5} P_{6} \partial \text { id }=0 .
\end{aligned}
$$

Now consider the general case. Let $\boldsymbol{y}=\left(y_{1}, \ldots, y_{r}\right)$ represent a critical point of the $s_{2 r+1}$ master function (11) associated with parameters $\boldsymbol{z}, \boldsymbol{\Lambda}, \Lambda_{\infty}$. Introduce the functions $\bar{y}_{1}, \ldots, \bar{y}_{r}$ by formula (15), where $B=\left(b_{i, j}\right)$ is the matrix inverse to the Cartan matrix of $s o_{2 r+1}$. Then the $s p_{2 r}$ Miura oper associated with $\boldsymbol{y}$ has the form

$$
D=\partial+\sum_{j=1}^{r} F_{j}+\sum_{j=1}^{r} \log ^{\prime}\left(\bar{y}_{j}\right) H_{j} .
$$

Let $\boldsymbol{u}=\left(u_{1}, \ldots, u_{2 r-1}\right)=\left(y_{1}, \ldots, y_{r}, \ldots, y_{1}\right)$ be the tuple representing the corresponding $s l_{2 r}$ critical point.

For $i=1, \ldots, r$, let $y_{i}^{[i]}, y_{i+1}^{[i, i+1]}, \ldots, y_{r}^{[i, \ldots, r]}$ be the $s o_{2 r+1}$ diagonal sequence of polynomials associated with $\boldsymbol{y}$ and the sequence of indices $[i, i+1, \ldots, r]$, in other words,

$$
\begin{gathered}
W\left(y_{i}, y_{i}^{[i]}\right)=T_{i} y_{i-1} y_{i+1}, \quad W\left(y_{i+1}, y_{i+1}^{[i, i+1]}\right)=T_{i+1} y_{i}^{[i]} y_{i+2}, \ldots, \\
W\left(y_{r-1}, y_{r-1}^{[i, \ldots, r-1]}\right)=T_{r-1} y_{r-2}^{[i, \ldots, r-2]} y_{r}, \quad W\left(y_{r}, y_{r}^{[i, \ldots, r]}\right)=T_{r}\left(y_{r-1}^{[i, \ldots, r-1]}\right)^{2} .
\end{gathered}
$$

For $i=1, \ldots, r-1$, let

$$
\begin{array}{rll}
u_{i}^{[i]}=y_{i}^{[i]}, \quad u_{i+1}^{[i, i+1]}=y_{i+1}^{[i, i+1]}, & \ldots, & u_{r-1}^{[i, \ldots, r-1]}=y_{r-1}^{[i, \ldots, r-1]}, \\
u_{r}^{[i, \ldots, r]}, \quad u_{r+1}^{[i, \ldots, r+1]}, & \ldots, & u_{2 r-i-1}^{[i, \ldots, 2 r-i-1]}
\end{array}
$$

be the $s l_{2 r}$ diagonal sequence of polynomials associated with $\boldsymbol{u}$ and the sequence of indices $[i, i+1, \ldots, 2 r-i-1]$, in other words,

$$
\begin{gathered}
W\left(y_{r}, u_{r}^{[i, \ldots, r]}\right)=T_{r} y_{r-1}^{[i, \ldots, r-1]} y_{r-1}, \quad W\left(y_{r-1}, u_{r+1}^{[i, \ldots, r+1]}\right)=T_{r-1} u_{r}^{[i, \ldots, r]} y_{r-2}, \\
W\left(y_{r-l}, u_{r+l}^{[i, \ldots, r+l]}\right)=T_{r-l} u_{r+l-1}^{[i, \ldots, r+l-1]} y_{r-l-1}, \quad \text { for } l=2, \ldots, r-i-1 .
\end{gathered}
$$


For $i \in\{1, \ldots, r\}$ set $F_{i, i}=F_{i}$. For $1 \leq i<j<r$ set

$$
F_{i, j}=\left[F_{j},\left[F_{j-1},\left[\ldots,\left[F_{i+1}, F_{i}\right] \ldots\right]\right]\right] \text {. }
$$

Set $F_{i, r}^{*}=\left[F_{r}, F_{i, r-1}\right]$ and for $1 \leq i<j<r$ set

$$
F_{i, j}^{*}=\left[F_{j},\left[F_{j+1},\left[\ldots\left[F_{r-2},\left[F_{r-1}, F_{i, r}^{*}\right]\right] \ldots\right]\right]\right] .
$$

Define $r+1$ functions $Y_{0}, Y_{1}, \ldots, Y_{r}$ of $x$ with values in $\operatorname{Sp}(2 r, \mathbb{C})$ by the formulas

$$
\begin{aligned}
& Y_{0}=\prod_{j=1}^{r} y_{j}{ }^{-H_{j}} T_{j}{ }^{w_{j}} \\
& Y_{i}=\left(\prod_{j=i}^{r-1} e^{\frac{y_{j}^{[i, \ldots, j]}}{y_{j}} F_{i, j}}\right) e^{\frac{1}{2} \frac{y_{r}^{[i, \ldots, r]}}{y_{r}}}\left[\left[F_{r}, F_{i, r-1}\right], F_{i, r-1}\right]\left(\prod_{j=r}^{2 r-i-1} e^{\frac{u_{j}^{[i, \ldots, j]}}{y_{2 r-j}} F_{i, 2 r-j}^{*}}\right)
\end{aligned}
$$

for $i \in\{1, \ldots, r-1\}$, and $Y_{r}=e^{\frac{y_{r}^{[r]}}{y_{r}} F_{r}}$.

Note that inside each product the factors commute.

Theorem 6.2. The product $Y_{0} Y_{1} \ldots Y_{r}$ is a solution of the differential equation $D Y=0$ with values in $\operatorname{Sp}(2 r, \mathbb{C})$ where $D$ is given by (21).

The proof is straightforward. One uses Lemma 6.3 and then shows that

$$
\left(\partial+\frac{\bar{y}_{r-1}^{2}}{\bar{y}_{r}{ }^{2}} F_{r}+\sum_{j=i}^{r-1} \frac{\bar{y}_{j-1} \bar{y}_{j+1}}{\bar{y}_{j}{ }^{2}} F_{j}\right) Y_{i}=Y_{i}\left(\partial+\frac{\bar{y}_{r-1}^{2}}{\bar{y}_{r}{ }^{2}} F_{r}+\sum_{j=i+1}^{r-1} \frac{\bar{y}_{j-1} \bar{y}_{j+1}}{\bar{y}_{j}{ }^{2}} F_{j}\right)
$$

for $i=1, \ldots, r-1$.

Remark. Theorems 6.1 and 6.2 give explicit formulas for solutions of the differential equation $D Y=0$ where $D$ is the Miura oper associated to a critical point of type $A_{r}$ or $B_{r}$. In a similar way one can construct explicit formulas for solutions in the case of the Miura oper associated to a critical point of type $C_{r}$, cf. Section 7 in [MV1].

6.4. General formulas for solutions. Let $\mathfrak{g}$ be a simple Lie algebra with Cartan matrix $A$. Let ${ }^{t} \mathfrak{g}$ be its Langlands dual with Chevalley generators $F_{1}, \ldots, F_{r}, H_{1}, \ldots, H_{r}$, $E_{1}, \ldots, E_{r}$. Let $w_{1}, \ldots, w_{r}$ be the fundamental coweights of ${ }^{t} \mathfrak{g}$. Let ${ }^{t} G$ be the complex simply connected Lie group with Lie algebra ${ }^{t} \mathfrak{g}$.

Let $V$ be a complex finite dimensional representation of ${ }^{t} G$. Let $v_{\text {low }}$ be a lowest weight vector of $V,{ }^{t} \mathfrak{n}_{-} v_{\text {low }}=0$.

Let $\boldsymbol{y}=\left(y_{1}, \ldots, y_{r}\right)$ represent a critical point of the $\mathfrak{g}$ master function (11) associated with parameters $\boldsymbol{z}, \boldsymbol{\Lambda}, \Lambda_{\infty}$. Let $D_{\boldsymbol{y}}$ be the ${ }^{t} \mathfrak{g}$ Miura oper associated with $\boldsymbol{y}$.

Let $\boldsymbol{i}=\left[i_{1}, \ldots, i_{k}\right], i_{j} \in\{1, \ldots, r\}$, be a sequence of natural numbers. Let $\boldsymbol{y}^{\left[i_{1}\right]}=$ $\left(y_{1}^{\left[i_{1}\right]}, \ldots, y_{r}^{\left[i_{1}\right]}\right), \boldsymbol{y}^{\left[i_{1}, i_{2}\right]}=\left(y_{1}^{\left[i_{1}, i_{2}\right]}, \ldots, y_{r}^{\left[i_{1}, i_{2}\right]}\right), \ldots, \boldsymbol{y}^{\left[i_{1}, \ldots, i_{k}\right]}=\left(y_{1}^{\left[i_{1}, \ldots, i_{k}\right]}, \ldots, y_{r}^{\left[i_{1}, \ldots, i_{k}\right]}\right)$ be a sequence of tuples associated with the critical point $\boldsymbol{y}$ and the sequence of indices $\boldsymbol{i}$, see Section 2.5. 
Theorem 6.3. The $V$-valued function

$$
\begin{aligned}
Y=\exp \left(-\log ^{\prime}\left(\frac{y_{i_{1}}^{\left[i_{1}\right]}}{y_{i_{1}}}\right) E_{i_{1}}\right) \exp \left(-\log ^{\prime}\left(\frac{y_{i_{2}}^{\left[i_{1}, i_{2}\right]}}{y_{i_{2}}^{\left[i_{1}\right]}}\right) E_{i_{2}}\right) \ldots \\
\exp \left(-\log ^{\prime}\left(\frac{y_{i_{k}}^{\left[i_{1}, \ldots, i_{k}\right]}}{y_{i_{k}}^{\left[i_{1}, \ldots, i_{k-1}\right]}}\right) E_{i_{k}}\right) \prod_{j=1}^{r}\left(y_{j}^{\left[i_{1}, \ldots, i_{k}\right]}\right)^{-H_{j}} T_{j}{ }^{w_{j}} v_{\text {low }}
\end{aligned}
$$

is a solution of the differential equation

$$
D_{\boldsymbol{y}} Y=0 \text {. }
$$

The proof is straightforward and follows from the identity

$$
D_{\boldsymbol{y}^{\left[i_{1}, \ldots, i_{j}\right]}}=\exp \left(\operatorname{ad} \log ^{\prime}\left(\frac{y_{i_{j}}^{\left[i_{1}, \ldots, i_{j}\right]}}{\left.y_{i_{j}}^{\left[i_{1}, \ldots, i_{j}\right]}\right]}\right) E_{i_{j}}\right) \cdot D_{\boldsymbol{y}^{\left[i_{1}, \ldots, i_{j-1}\right]}}
$$

see Theorem 3.1 .

Let $d$ be the determinant of the Cartan matrix of $\mathfrak{g}$.

Corollary 6.5. Every coordinate of every solution of the equation $D_{\boldsymbol{y}} Y=0$ with values in a finite dimensional representation of ${ }^{t} G$ can be written as a rational function $R\left(f_{1}, \ldots, f_{N} ; T_{1}^{1 / d}, \ldots, T_{r}^{1 / d}\right)$ of functions $T_{1}^{1 / d}, \ldots, T_{r}^{1 / d}$ and suitable polynomials $f_{1}, \ldots, f_{N}$ which appear as coordinates of tuples in the $\mathfrak{g}$ population $P_{\boldsymbol{y}}$ originated at $\boldsymbol{y}$.

Since ${ }^{t} G$ has a faithful finite dimensional representation, the solutions of the differential equation $D_{\boldsymbol{y}} Y=0$ with values in ${ }^{t} G$ also can be written as rational functions of functions $T_{1}^{1 / d}, \ldots, T_{r}^{1 / d}$ and coordinates of tuples of $P_{\boldsymbol{y}}$, cf. Sections 6.2 and 6.3 .

\section{REFERENCES}

[BD] A. Beilinson and V. Drinfeld, Opers, preprint.

[BGG] I.N. Bernshtein, I.M. Gel'fand, and S.I. Gel'fand, Structure of representations generated by vectors of highest weight, Funct. Anal. Appl. 5 (1971), 1-8.

[B] A. Borel, Linear algebraic groups, W. A. Benjamin, 1969.

[BM] L. Borisov and E. Mukhin, Self-self-dual spaces of polynomials, math.QA/0308128 (2003), 1-38.

[DS] V. Drinfeld and V. Sokolov, Lie algebras and KdV type equations, J. Sov. Math. 30 (1985), 19752036.

[FFR] B. Feigin, E. Frenkel, and N. Reshetikhin, Gaudin model, Bethe Ansatz and Critical Level, Commun. Math. Phys. 166 (1994),29-62.

[F1] E. Frenkel, Affine Algebras, Langlands Duality and Bethe Ansatz, math.QA/9506003 (1999), 1-34.

[F2] E. Frenkel, Opers on the projective line, flag manifolds and Bethe anzatz. math.QA/0308269 (2003), 1-48.

[H] J. Humphreys, Linear algebraic groups, Springer-Verlag, 1975.

$[\mathrm{K}] \quad$ V. Kac, Infinite-dimensional Lie algebras, Cambridge University Press, 1990.

[MV1] E. Mukhin and A. Varchenko, Critical Points of Master Functions and Flag Varieties, math.QA/0209017 (2002), 1-49. 
[MV2] E. Mukhin and A. Varchenko, Populations of solutions of the XXX Bethe equations associated to Kac-Moody algebras, math.QA/0212092 (2002), 1-8.

[MV3] E. Mukhin and A. Varchenko, Solutions to the XXX type Bethe Ansatz equations and flag varieties, math.QA/0211321 (2002), 1-32.

[MV4] E. Mukhin and A. Varchenko, Discrete Miura Opers and Solutions of the Bethe Ansatz Equations, math.QA/0401137 (2004), 1-26.

[RV] N. Reshetikhin, A. Varchenko, Quasiclassical asymptotics of solutions to the KZ equations, Geometry, topology \& physics, Conf. Proc. Lecture Notes Geom. Topology, VI, Internat. Press, Cambridge, MA (1995), 293-322.

[ScV] I. Scherbak and A. Varchenko, Critical point of functions, sl $l_{2}$ representations and Fuchsian differential equations with only univalued solutions, math. QA/0112269, (2001) 1-25.

[SV] V. Schechtman and A. Varchenko, Arrangements of hyperplanes and Lie algebra homology, Invent. Math., 106 (1991), 139-194. 